\title{
Sitios prioritarios para la conservación de la riqueza florística y el endemismo de la Sierra Norte de Oaxaca, México
}

\section{Priority sites for the conservation of floristic richness and endemism of the Northern Sierra of Oaxaca, Mexico}

\author{
Mario Ernesto Suárez-Motal,3 (D), José Luis Villaseñor² (D), Marleny B. Ramírez-Aguirrel
}

1 Universidad de la Sierra Juárez, Avenida Universidad s.n., 68725 Ixtlán de Juárez, Oaxaca, México.

2 Universidad Nacional Autónoma de México, Instituto de Biología, Departamento de Botánica, Apdo. postal 70-233, 04510 Cd. Mx., México.

3 Autor para la correspondencia: suarezmota.mario@gmail.com

Recibido: 6 de octubre de 2017. Revisado: 15 de noviembre de 2017. Aceptado: 11 de enero de 2018. Primero en línea: 29 de mayo de 2018. Publicado: 1 de julio de 2018.

\section{Citar como:}

Suárez-Mota, M. E., J. L. Villaseñor y M. B. Ramírez-Aguirre. 2018. Sitios prioritarios para la conservación de la riqueza florística y el endemismo de la Sierra Norte de Oaxaca, México. Acta Botanica Mexicana 124: 49-74. DOI: 10.21829/ abml24.2018.1296

DOI:

10.21829/abm124.2018.1296

\section{Resumen:}

Antecedentes y Objetivos: Una de las principales estrategias para la protección de la diversidad biológica es el establecimiento de sistemas de áreas para su conservación, cuyo manejo minimice los riesgos de extinción. Actualmente, en México las áreas protegidas cubren aproximadamente $12 \%$ de la superficie del país. No obstante, es necesario incrementar los esfuerzos de conservación de la diversidad vegetal, especialmente en las altitudes medias de las zonas montañosas del país. Oaxaca es uno de los estados con mayor diversidad biológica y endemismos; sin embargo, las áreas naturales protegidas que se encuentran decretadas en el estado no son suficientes para conservar su riqueza biológica. Este estudio se plantea con el objetivo de definir una red de áreas de conservación en la Sierra Norte del estado de Oaxaca, región que es reconocida por su biodiversidad. Es una zona de amplio aprovechamiento forestal y a la fecha no existen áreas decretadas para la conservación de su biodiversidad.

Métodos: Usando datos climáticos y la información sobre registros de presencia de las especies de la familia Asteraceae, se elaboraron modelos de nicho ecológico para determinar patrones de riqueza e identificar sitios prioritarios de conservación.

Resultados: Se identifica una red de sitios prioritarios para la conservación, dentro de las zonas con aprovechamiento forestal, que ayudarían en la conservación de la riqueza florística del estado.

Conclusiones: Los resultados indican las áreas importantes donde se podrían plantear estrategias de conservación en la Sierra Norte de Oaxaca. El uso de especies de Asteraceae como subrogados de la biodiversidad funcionaría como eje para concentrar futuros esfuerzos de inventario y de protección de la riqueza florística en tales sitios. La Red de Áreas de Conservación estructurada en este trabajo permitirá en un futuro detectar y seleccionar, con bases conceptuales más sólidas, áreas que se integren al Sistema Nacional de Áreas Protegidas.

Palabras clave: Asteraceae, complementariedad, ConsNet, red de áreas de conservación.

\section{ABSTRACT:}

Background and Aims: One of the main strategies for the protection of biological diversity is the establishment of systems of areas for conservation, whose management minimizes the risks of extinction. Currently, in Mexico, protected areas cover approximately $12 \%$ of the country's surface. However, there is a need to increase efforts to conserve plant diversity, especially at the middle altitudes of the mountainous areas of the country. Oaxaca is one of the states with greater biological diversity and endemisms; however, the natural areas that are decreed in the state are not sufficient to conserve their biological richness. This study aims to define a network of conservation areas in the Sierra Norte of the state of Oaxaca, a region that is recognized for its great biodiversity. The Sierra Norte is an area of extensive forestry use and to date there are no areas decreed for the conservation of its biodiversity.

Methods: Using climatic data and information about presence records of the species of the Asteraceae family, ecological niche models were developed to determine patterns of richness and to identify priority conservation sites.

Results: The results identify a network of priority conservation sites within the forestry zones that would assist in the conservation of its great floristic richness.

Conclusions: The important areas where conservation strategies could be proposed in the Sierra Norte de Oaxaca are identified. The use of species of Asteraceae as surrogates of biodiversity would work as an axis to concentrate future efforts of inventory and protection of floristic richness in such sites. The Network of Conservation Areas structured in this work will allow in the future to detect and select, with more solid conceptual bases, areas that can be integrated into the National System of Protected Areas.

Key words: Asteraceae, complementarity, ConsNet, network of conservation areas. 


\section{INTRODUCCIÓN}

México tiene una vasta riqueza cultural y biológica enmarcada dentro de una compleja geografía. Su ubicación territorial le permite abarcar prácticamente todos los tipos de ecosistemas conocidos a nivel mundial, desde zonas desérticas hasta bosques y selvas exuberantes, cubriendo cañadas, costas y sierras. Por esta razón se le ha considerado entre los 10 países más ricos del mundo, tanto por las especies de animales como por las de vegetales que lo conforman (Narro, 2010).

La biodiversidad, formada a lo largo de millones de años, provee la materia prima de la evolución; en consecuencia, toda ella debería de ser conservada (Margules y Sarkar, 2009). México, junto con Centroamérica, constituye una región de alta riqueza de plantas, donde el nivel de endemismo, tanto a nivel genérico como específico, es alto (Rzedowski, 1998; Myers et al., 2000; Villaseñor, 2016). Se estima que originalmente $52 \%$ del país estaba cubierto con bosques y selvas (Rzedowski y Reyna-Trujillo, 1990); sin embargo, los datos del Inventario Forestal Nacional 2000 reportan que dicha superficie se había reducido a 33\% del territorio nacional (Palacio-Prieto et al., 2000; Ricker et al., 2007). Debido a su fragilidad y a la, actualmente, reducida cobertura de los diferentes tipos de vegetación; se ha discutido que México requiere acciones más eficientes para la protección y conservación de su biodiversidad (Koleff et al., 2007). Aunque no existen áreas de conservación con decreto federal o estatal, en algunas localidades de México las comunidades han planteado el establecimiento de "Zonas de conservación indígena-comunitarias" (ICCAs) (Robson, 2007; Martin et al., 2011).

El establecimiento de sistemas de áreas para la conservación, cuyo manejo minimice los riesgos de extinción, es fundamental para la protección de la diversidad biológica (Margules y Sarkar, 2009). Para la identificación de dichas áreas prioritarias, se ha propuesto la planeación sistemática de la conservación utilizando protocolos específicos para identificarlas y desvincularlas de los procesos que amenazan su permanencia (Margules y Sarkar, 2009). De esta manera, los sistemas de áreas prioritarias en cada región del mundo deberían constituir el marco en el que se puedan construir las acciones de conservación (Margules y Sarkar, 2009). Actualmente, en México las áreas protegidas cubren aproximadamente $12 \%$ de la superficie del país; sin embargo, es necesario incrementar los esfuerzos de conservación de la diversidad vegetal, especialmente en las altitudes medias de las zonas montañosas del país (EMCV, 2008).

La flora fanerogámica de México es una de las más diversas del planeta. El recuento más reciente reporta 23,314 especies; la mitad de ellas son endémicas (Villaseñor, 2016). Estos datos sugieren que el territorio nacional representa una región donde se han originado y evolucionado un gran número de linajes vegetales (Rzedowski, 1998). Por el número de endemismos presentes y por su gran riqueza florística, uno de los estados que destacan en México es Oaxaca (García-Mendoza, 2004; Suárez-Mota y Villaseñor, 2011; Villaseñor y Ortiz, 2012; Villaseñor, 2016). La cifra más reciente sobre su flora reporta 10,229 especies, de las cuales 760 son endémicas del estado (Villaseñor, 2016). Algunas áreas de Oaxaca son particularmente importantes por su riqueza y el número de endemismos que contienen. Lorence y García-Mendoza (1989) señalan como ejemplo la Sierra Norte. Ésta representa el límite septentrional de distribución de muchos taxones mesoamericanos de montaña y es una región de importancia para las pteridofitas y géneros como Begonia Hook. f., Miconia Ruiz \& Pav., Piper L. o Quercus L. La Sierra cuenta con un inventario florístico (Torres-Colín et al., 2009), donde se reconocen 830 géneros y 2160 especies de plantas vasculares para la región de Ixtlán y algunas localidades colindantes (región que se conoce como Sierra de Juárez). En dicho inventario se reporta que la flora se distribuye en 10 tipos diferentes de vegetación, de los 26 reportados en el estado de Oaxaca cuando se realizó este trabajo (Torres-Colín et al., 2009). Asteraceae constituye la familia más importante por su riqueza de especies (174) y es una de las 34 familias que concentran $66.3 \%$ de toda la riqueza florística de la Sierra (Torres-Colín et al., 2009). 
La familia Asteraceae ocupa un lugar preponderante en la flora de México, tanto por su número de géneros como de especies pues contribuye sustancialmente a la riqueza florística nacional con 362 géneros y más de 3000 especies (Balleza y Villaseñor, 2002; Villaseñor, 1993, 2004, 2016); además, se distribuye en todos los tipos de vegetación existentes en el país. Por otra parte, estudios recientes señalan que la familia puede ser un buen subrogado de la riqueza florística total (Villaseñor et al., 2005, 2007; Suárez-Mota et al., 2015, 2017). Oaxaca es el estado mexicano con la mayor riqueza de ésta familia botánica; su número asciende a más de 800 especies, de las cuales poco más de 500 son endémicas de México y unas 133 son endémicas del estado (Villaseñor, 2004; SuárezMota y Villaseñor, 2011).

Una evaluación reciente (Villaseñor, datos no publicados) revela la existencia de 2704 especies nativas de plantas vasculares en la Sierra Norte. Esta cifra es mayor en $25 \%$ con respecto a lo reportado por Torres-Colín y colaboradores en 2009, quienes se concentraron exclusivamente en el Distrito de Ixtlán. Hay que destacar que la riqueza no está balanceada entre los tres distritos administrativos que conforman el territorio político de la Sierra (Ixtlán, Mixe y Villa Alta; Cuadro 1); seguramente el mayor esfuerzo de recolecta en el Distrito de Ixtlán ha generado la notable diferencia en sus valores de riqueza.

Para la selección de áreas de conservación de diversas especies y determinación de patrones de distribución, en la última década, una de las estrategias usadas ha sido utilizar modelos de nicho ecológico (MNE). Los MNE son aproximaciones empíricas o matemáticas al nicho ecológico de una especie, que se emplean como sustitutos de la biodiversidad. Con ello se persigue el objetivo de relacionar diferentes tipos de variables ecogeográficas con la distribución de las especies y así identificar las variables que limitan y definen su nicho (Guisan y Zimmermann, 2000; Peterson et al., 2002). Estos MNE están basados en el concepto de nicho ecológico de Hutchinson (1957), que relaciona la información biológica con la ambiental, identificando las zonas donde no existan registros previos de la especie, corrigiendo la predicción y obteniendo su área de distribución potencial (Guisan y Zimmermann, 2000; Peterson et al., 2002; Suárez-Mota et al., 2015). El resultado final de un MNE puede ser una representación espacial de los hábitats favorables para la presencia de una especie, inclusive en áreas poco muestreadas o previendo cambios futuros en el entorno (Shugart, 1990; Sykes et al., 1996; Guisan y Zimmermann, 2000;

Cuadro 1: Riqueza de especies vasculares y de la familia Asteraceae en la Sierra Norte de Oaxaca, México y su distribución en los tres distritos que la conforman (Ixtlán, Mixe y Villa Alta).

\begin{tabular}{lcccc}
\hline & Sierra Norte & Distrito Ixtlán & Distrito Mixe & Distrito Villa Alta \\
\cline { 2 - 5 } Riqueza total & 2704 & 2500 & 657 & 376 \\
\hline Especies nativas & 744 & 683 & 145 & 55 \\
Endémicas de México & 141 & 126 & 26 & 13 \\
Endémicas de Oaxaca & 87 & 72 & 18 & 9 \\
Endémicas Sierra Norte & & & & 36 \\
\hline Familia Asteraceae & 340 & 297 & 179 & 11 \\
\hline Especies nativas & 147 & 136 & 57 & 2 \\
Endémicas de México & 22 & 22 & 4 & 1 \\
Endémicas de Oaxaca & 11 & 11 & 1 & \\
Endémicas Sierra Norte & & & & \\
\hline
\end{tabular}


Teixeira y Arntzen, 2002; Araújo et al., 2006; Engler et al., 2009). También son útiles para evaluar las tendencias de abundancia de las especies o la posibilidad de persistencia en determinadas áreas (Araújo y Williams, 2000; Real et al., 2009).

La modelación de la distribución geográfica de las especies permite identificar patrones generales de biodiversidad, lo que facilita decidir acerca de las prioridades de conservación a gran escala (Chapman y Busby, 1994; Franklin, 1995; Austin, 1998, 2002; Guisan y Zimmermann, 2000; Elith y Burgman, 2002; Scott et al., 2002). Además, ha demostrado su importancia en el establecimiento de prioridades para la conservación de la biodiversidad, mediante la identificación de áreas particularmente especiales para tales estrategias (Ceballos et al., 2005).

Un factor importante que afecta o determina la distribución de los organismos es el clima; por consiguiente, los análisis de las variables climáticas ayudan a entender por qué una especie crece en un determinado sitio y no en otro (Lindenmayer et al., 1996). Las plantas adaptadas a un régimen climático particular con frecuencia tienen morfologías similares o ciertos tipos de formas de crecimiento (Cain, 1950). Se han utilizado los elementos del clima para delimitar grandes unidades de vegetación, como es el caso del sistema de las zonas de vida elaborado por Holdridge (1967), o bien, para relacionar las adaptaciones de los grandes biomas con ciertos intervalos de variables ambientales (Cox et al., 1976). De manera particular, cada especie tiene su propio perfil climático, por lo que el análisis de las variables que lo determinan, puede servir para cuantificar las diferencias en sus áreas de distribución. En otras palabras, esto ayuda a determinar el espacio climático en el cual se considera que una especie vegetal sobrevive bajo condiciones naturales, siendo diferente entre las especies (Fischer et al., 2001).

Busby (1986) y Nix (1986) desarrollaron un conjunto de variables climáticas que pueden ser significativas para entender la distribución de una amplia gama de la biota. Un factor clave en este enfoque es que las variables ambientales son más simples y más fáciles de definir que otras que son incluidas en el inventario de la biota a nivel regional o nacional. Para la conservación biológica a gran escala, tal enfoque medioambiental sin duda podría favorecer el establecimiento de reservas para un número cada vez mayor de especies raras y/o en peligro de extinción.

En la Sierra Norte de Oaxaca, también conocida como Sierra Madre de Oaxaca o Sierra de Juárez, los ecosistemas templados están conformados principalmente por bosques húmedos de montaña o de pino-encino, siendo de gran importancia ecológica y silvícola (VásquezCortés, 2013). En esta región la mayor parte de las comunidades rurales practican el aprovechamiento forestal, enfocados principalmente hacia la producción de madera (Castellanos-Bolaños et al., 2010). Sin embargo, dicha actividad ejerce un efecto negativo sobre la dinámica de la vegetación y sus componentes florísticos (CastellanosBolaños et al., 2010). Para ayudar a entender el papel de la riqueza florística en la composición regional y su relevancia en la conservación de los bosques de la Sierra Norte, este trabajo se plantea el objetivo de definir una red de áreas de conservación biológica. Para ello, se utiliza una estrategia de análisis de complementariedad, utilizando la riqueza conocida y modelos de nicho ecológico de especies endémicas de Asteraceae, utilizándolas como substitutos de la biodiversidad de la región. La hipótesis de este trabajo es que sitios con alta riqueza de especies de Asteraceae serán igualmente sitios de alta riqueza de especies de otros grupos vegetales.

\section{Materiales y Métodos}

\section{Área de estudio}

La Sierra Norte o Sierra Madre de Oaxaca es una de las ocho grandes regiones que integran el estado de Oaxaca, comprende un territorio de gran riqueza forestal (GarcíaMendoza, 2004). Se ubica dentro de las coordenadas $16^{\circ} 58^{\prime}$ y $17^{\circ} 48^{\prime}$ de latitud $\mathrm{N}$ y $95^{\circ} 8^{\prime}$ y $96^{\circ} 47^{\prime}$ de longitud W e incluye las regiones de La Chinantla, Sierra Mazateca, Sierra Mixe y Sierra de Juárez. En el concepto de territorio político; cuenta con 68 municipios divididos en tres distritos: Ixtlán, Mixe y Villa Alta (Fig. 1). Es una región importante por su compleja fisiografía que incluye 34 tipos de 


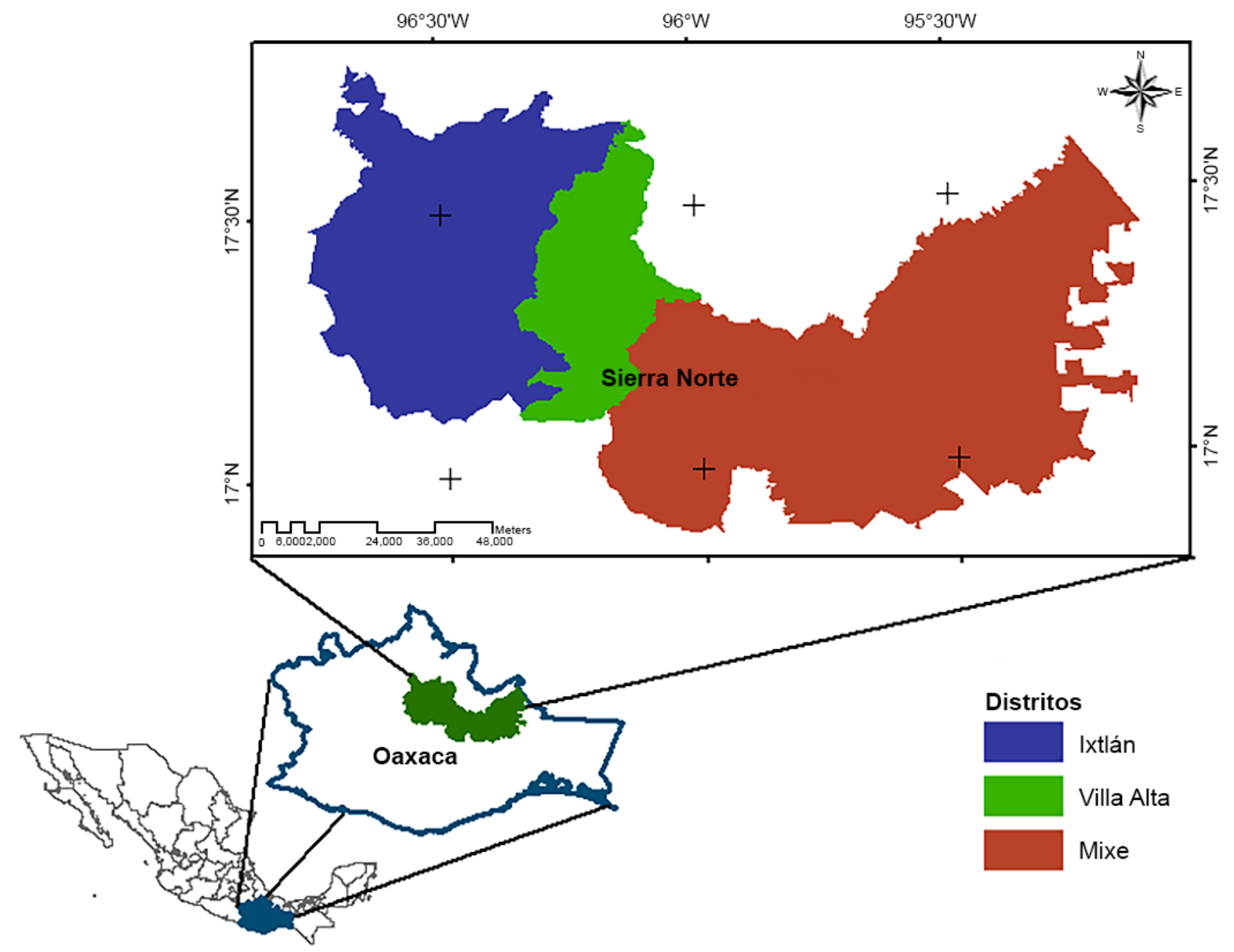

Figura 1: Ubicación geográfica de la Sierra Norte de Oaxaca, México y sus distritos (Ixtlán, Mixe y Villa Alta).

vegetación diferentes (INEGI, 2015), 19 de suelos (INEGI, 2004b), 28 de rocas (INEGI, 2005), 19 de clima (INEGI, 2004a) y los cinco principales tipos de biomas (Villaseñor y Ortiz, 2014). La superficie que comprende esta región estimada en este trabajo es de aproximadamente $8925.7 \mathrm{~km}^{2}$.

\section{Obtención de datos}

Los datos de las especies endémicas de Oaxaca y de la Sierra Norte fueron obtenidos de dos fuentes principales: 1) consulta de ejemplares herborizados, resguardados en diferentes herbarios, tanto nacionales (ENCB, IZTA, MEXU, XAL) como extranjeros (ARIZ, K, LL, TEX) y
2) revisión de los registros de bases de datos disponibles en la Red Mundial de Información sobre Biodiversidad (REMIB, 2017) de la Comisión Nacional para el Conocimiento y Uso de la Biodiversidad (CONABIO), la Unidad de Informática para la Biodiversidad (UNIBIO, 2017) del Instituto de Biología de la Universidad Nacional Autónoma de México y la Global Biodiversity Information Facility (GBIF, 2017). Estos datos se evaluaron para verificar su correcta determinación y georreferenciación; varias especies fueron además verificadas directamente con trabajo de campo, complementando así la información sobre su distribución geográfica en la región. 


\section{Modelos de nicho ecológico}

El mapa que delimita la zona de la Sierra Norte se sobrepuso sobre un modelo digital de elevación (MDE) con una resolución de $120 \mathrm{~m}$. Este mapa se utilizó como base para realizar modelos de nicho ecológico de las especies seleccionadas (Apéndice). Se utilizaron los programas MaxEnt 3.3.3k (Phillips y Dudík, 2008) y el sistema de información geográfica (SIG) ArcMap 10.1 (ESRI, 2011) para la elaboración y edición de los mapas que muestran los nichos ecológicos de las especies. Siguiendo los criterios del diagrama BAM para la elaboración de modelos de nicho, propuesto por Soberón y Peterson (2005), la región geográfica (M del diagrama) para la elaboración de los modelos se definió con los límites de la Sierra Norte (Fig. 1). Las variables climáticas utilizadas para la generación de los modelos se obtuvieron de Cuervo-Robayo et al. (2013; Cuadro 2).

Los datos geográficos que señalan la distribución de las especies, junto con las variables climáticas, se incorporaron al programa MaxEnt para generar los modelos de nicho ecológico (MNE). Para las especies que contaron con 25 registros o más, se empleó $75 \%$ de ellos como datos de entrenamiento y $25 \%$ restante como datos de prueba; los modelos de las especies con menos de 25 registros se obtuvieron usando $100 \%$ de los datos para entrenamiento. Se empleó el método del área bajo la curva (AUC, por sus siglas en inglés) de la Característica Operativa del Receptor (ROC por sus siglas en inglés) parcial como método de evaluación de los modelos. Finalmente el archivo tipo ASCII, generado por MaxEnt, fue importado como un mapa al Sistema de Información Geográfica (SIG) para su edición en modo binario.

\section{Selección de áreas prioritarias de conservación}

Los mapas obtenidos con los MNE se sobrepusieron con el mapa de la Sierra Norte. La coincidencia de esta sobreposición permitió elaborar una matriz de datos binarios para hacer el análisis de complementariedad mediante el uso de los algoritmos metaheurísticos, implementados en el software ConsNet (Ciarleglio et al., 2008, 2009). Con el uso de estos algoritmos se asegura la representatividad
Cuadro 2: Parámetros climáticos usados para generar los modelos de distribución potencial de las especies endémicas distribuidas en la Sierra Norte de Oaxaca, México.

1. Temperatura media anual $\left({ }^{\circ} \mathrm{C}\right)$.

2. Rango medio diurno $\left({ }^{\circ} \mathrm{C}\right)$.

3. Isotermalidad $\left({ }^{\circ} \mathrm{C}\right)$.

4. Temperatura estacional (\%).

5. Temperatura máxima del mes más cálido $\left({ }^{\circ} \mathrm{C}\right)$.

6. Temperatura mínima del mes más frío $\left({ }^{\circ} \mathrm{C}\right)$.

7. Rango de temperatura anual $\left({ }^{\circ} \mathrm{C}\right)$.

8. Temperatura media del trimestre más húmedo $\left({ }^{\circ} \mathrm{C}\right)$.

9. Temperatura media del trimestre más seco $\left({ }^{\circ} \mathrm{C}\right)$.

10. Temperatura media del trimestre más cálido $\left({ }^{\circ} \mathrm{C}\right)$.

11. Temperatura media del trimestre más frío $\left({ }^{\circ} \mathrm{C}\right)$.

12. Precipitación anual (mm).

13. Precipitación del mes más húmedo $(\mathrm{mm})$.

14. Precipitación del mes más seco (mm).

15. Precipitación estacional (C of V) (\%).

16. Precipitación del trimestre más húmedo $(\mathrm{mm})$.

17. Precipitación del trimestre más seco $(\mathrm{mm})$.

18. Precipitación del trimestre más cálido $(\mathrm{mm})$.

19. Precipitación del trimestre más frío (mm).

de los objetos de conservación, que en este caso son considerados subrogados o indicadores de la biodiversidad (especies y ecosistemas) en ambientes donde aún existen hábitats naturales. Los criterios de configuración espacial (área, forma y conectividad) forman parte del proceso de la planeación sistemática para definir redes de áreas de conservación haciendo uso de ConsNet (Ciarleglio et al., 2008).

Se evaluaron tres escenarios, definidos con base en tres algoritmos implementados en ConsNet: 1) selección de celdas con los substitutos de mayor déficit de representación (MDS2 por sus siglas en inglés), 2) ponderación de la rareza en primer lugar (RF4 por sus siglas en inglés), donde las celdas con mayor rareza de substitutos son elegidos con la máxima prioridad y 3) utilización de 
un algoritmo que combina los dos anteriores, denominado "InterLeaVes" (ILV4 por sus siglas en inglés) (Ciarleglio et al., 2009). Mediante la probabilidad de la distribución de especies en cada celda utilizada en el análisis, ConsNet hace una decisión de tipo binario (para seleccionar o no una celda sometida a un plan de conservación) y posteriormente ordena cada celda jerárquicamente, con base en su valor de biodiversidad (riqueza de especies). En este análisis se planteó un objetivo que redujo el número de celdas seleccionadas, maximizando la contigüidad de la red de áreas de conservación (RAC).

El número de tipos de vegetación y de uso de suelo, obtenidos de INEGI (2015), distribuidos en la Sierra Norte, fueron agrupados para definir los biomas presentes en la región de estudio, siguiendo la clasificación propuesta por Suárez-Mota y Villaseñor (2011) y Villaseñor y Ortiz (2012). Con los biomas así definidos se analizó la distribución de las especies y su correspondencia con las áreas de conservación identificadas en el análisis (Suárez-Mota et al., 2015).

\section{Resultados}

\section{Obtención de datos}

La familia Asteraceae registra en la Sierra Norte de Oaxaca 340 especies (Apéndice); 147 de ellas son endémicas de México, 22 endémicas del estado de Oaxaca y 11 de estas últimas solamente se conocen de la Sierra Norte. Aunque existe una red de áreas comunitarias protegidas, el Sistema Nacional de Áreas Naturales Protegidas (SINAP) no registra áreas protegidas federales en la Sierra; por consiguiente, ninguno de esos endemismos cuenta actualmente con una estrategia de protección o conservación (SINAP, 2014).

\section{Modelos de Nicho Ecológico}

El conocimiento sobre la distribución geográfica de las especies en la Sierra Norte es precario, solamente 138 (40.5\%) de las especies de Asteraceae cuentan con cinco o más registros (sitios diferentes de recolecta), el número mínimo que se recomienda para obtener los modelos de nicho ecológico utilizando MaxEnt (Phillips y Dudík,
2008). Un total de 142 especies representan las más raras de la flora, pues se conocen de la región de estudio únicamente con base en uno (95) o dos (46) sitios de recolecta (Apéndice). El modelo digital de elevación, con resolución de 120 m por pixel generó 614,007 celdas, cubriendo un área total de $8925.7 \mathrm{~km}^{2}$. Las celdas $\left(0.0144 \mathrm{~km}^{2}\right)$ donde se distribuyen las especies conocidas a partir de un solo registro se seleccionaron como sitios irremplazables para la conservación, pues contienen especies restringidas a porciones específicas de la Sierra. Por otra parte, el mayor número de ocurrencias y de especies se encontró en el intervalo altitudinal comprendido entre 2000 y $2400 \mathrm{~m}$.

La riqueza de especies por celdas varió entre 1 y 79. Es decir, se identificó una celda donde se concentran 79 taxa endémicos, lo que la ubica como la más importante, pues en un área tan reducida se concentra $23 \%$ del total de Asteraceae analizadas. Se encontró que Stevia microchaeta Sch. Bip. es la especie más ampliamente distribuida, registrada en 46 celdas, seguida de Ageratina ligustrina (DC.) R.M. King \& H. Rob. (en 26), las otras especies se distribuyen en un número menor de celdas. Los modelos de distribución potencial de estas dos especies sugieren que ocupan áreas de $2349.3 \mathrm{~km}^{2}$ (163) y $2205.55 \mathrm{~km}^{2}$ (153 celdas) respectivamente (Fig. 2).

\section{Selección de áreas prioritarias de conservación}

El Cuadro 3 muestra los resultados del uso de los modelos de distribución potencial y el programa ConsNet para generar la red de áreas de conservación (RAC). Se muestra el número de celdas obtenido con los tres algoritmos implementados en el software, empleando como meta de conservación $10 \%$ de las poblaciones de cada especie (suponiendo que cada celda contiene al menos una población de las especies allí registradas o estimadas), estrategia seguida en otros trabajos (p. ej., Suárez-Mota y Téllez-Valdés, 2014; Suárez-Mota et al., 2015). En general, la superficie obtenida con cada estrategia es bastante similar; sin embargo, el algoritmo MSD2 requiere menos superficie por las celdas que selecciona para la estrategia de conservación y menor perímetro que ocupan los polígonos que conforman su red de áreas de conservación. Por tal razón dicha estrategia es 


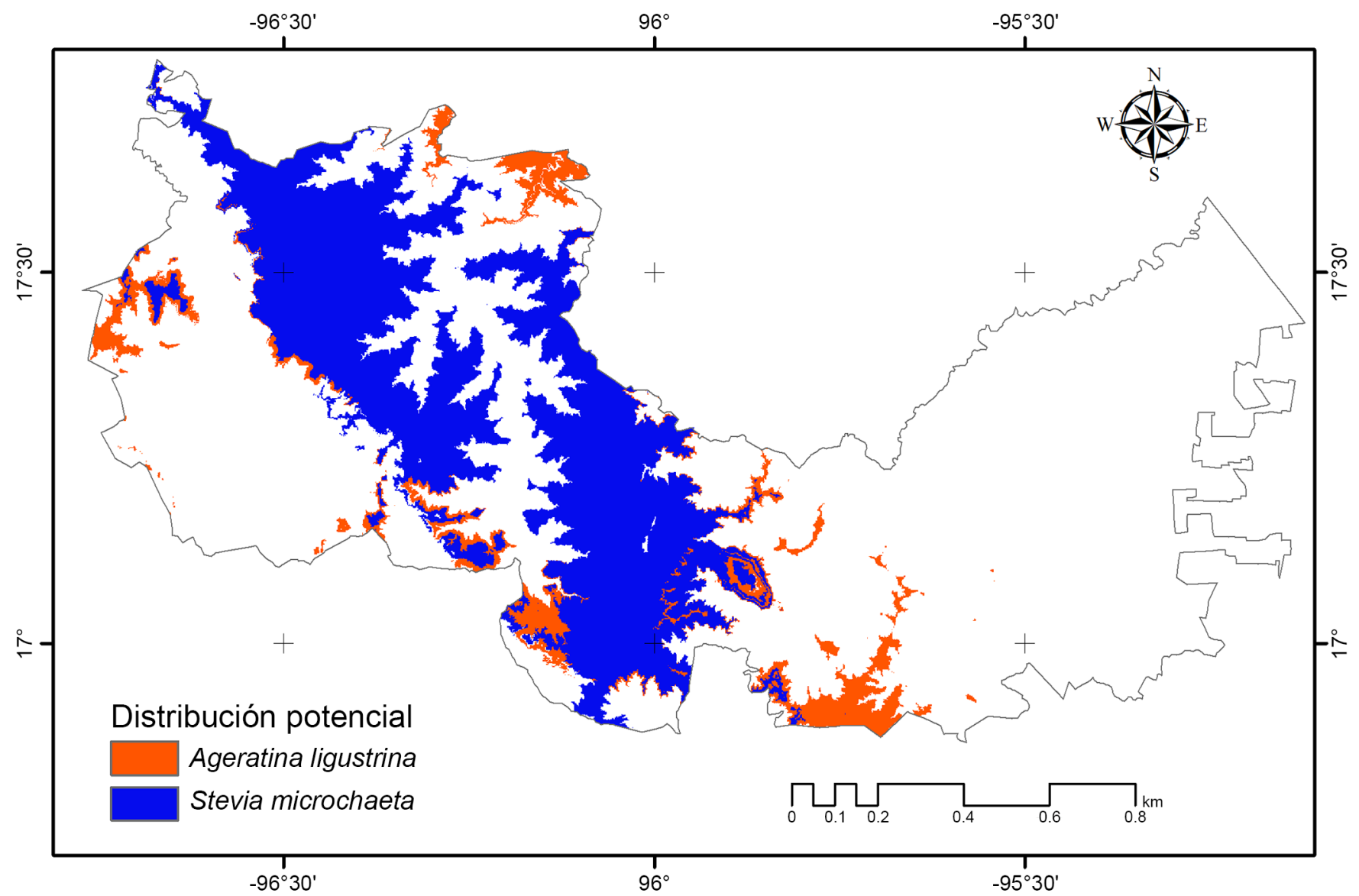

Figura 2: Modelos de distribución potencial de las especies con mayor área de distribución geográfica en la Sierra Norte de Oaxaca, México. La zona en color azul corresponde al área potencial de Stevia microchaeta Sch. Bip. compartida con Ageratina ligustrina (DC.) R.M. King \& H. Rob., mientras que la zona en color rojo señala las áreas de no coincidencia entre ambos modelos.

Cuadro 3: Resultados del análisis de complementariedad con los datos de Asteraceae de la Sierra Norte de Oaxaca, México, obtenidos con los algoritmos implementados en ConsNet. MDS2=substitutos de mayor déficit de representación; RF4=ponderando primero la rareza; ILV4=Combina los dos algoritmos anteriores.

\begin{tabular}{lccc}
\hline Algoritmo de búsqueda & $\begin{array}{c}\text { Total de } \\
\text { celdas }\end{array}$ & Área $\left(\mathrm{km}^{2}\right)$ & Perímetro $\left(\mathrm{km}^{2}\right)$ \\
& 59351 & 865.091 & 2864.531 \\
(MDS2 adyacencia) & 59352 & 865.105 & 2977.707 \\
(RF4 adyacencia) & 59547 & 867.917 & 3231.299 \\
(RF4) & 59548 & 867.932 & 3335.204 \\
(ILV4 adyacencia) & 59547 & 867.917 & 3231.299 \\
(ILV4) & 59548 & 867.932 & 3335.204 \\
\hline
\end{tabular}

la seleccionada para ilustrar los resultados (Fig. 3A); en total, la RAC incluye 147 polígonos, que varían desde 0.049 $\mathrm{km}^{2}$ el más pequeño hasta $490.58 \mathrm{~km}^{2}$ el más amplio.

La RAC mostrada en la figura $3 \mathrm{~A}$ incluye 30 de las 93 celdas consideradas como irremplazables. Comprende también 59,352 celdas, que representan $9.7 \%$ del total en que se dividió la zona de estudio. En dicha región se registran 225 especies $(65.8 \%)$ de 340 conocidas en toda la Sierra Norte (Apéndice). La prioridad de conservación considerando el complemento identifica ocho polígonos de esta RAC donde se podrían conservar poblaciones de estas 225 especies de Asteraceae de la Sierra Norte.

Los ocho polígonos identificados en la RAC de la figura 3B se nombraron de acuerdo con el Distrito don- 

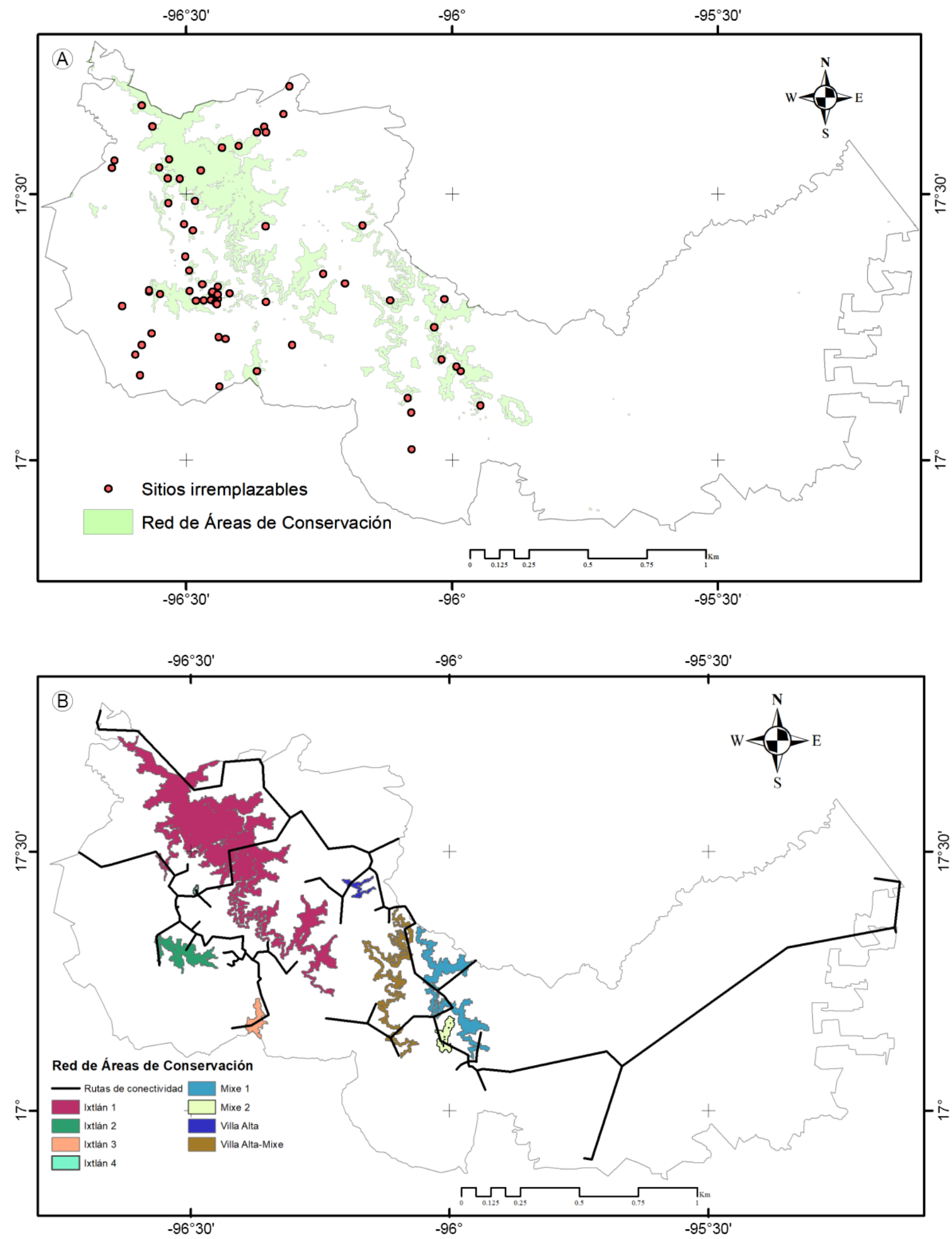

Figura 3: A. red de Áreas Prioritarias de conservación en la Sierra Norte de Oaxaca, México. Los puntos rojos indican los sitios irremplazables por contener especies conocidas solamente en dichas localidades aunque no coincidan con los polígonos que forman parte de la red; B. rutas de conectividad (mínima distancia) entre las áreas prioritarias de conservación en la Sierra Norte. Los nombres de los polígonos corresponden a las áreas complementarias del Cuadro 4 . 
de se ubican, numerándolos en orden descendente por la superficie que abarcan (Cuadro 4). De esta manera, Ixtlán I es el polígono que más especies registra (144) en una superficie que abarca $490.58 \mathrm{~km}^{2}$. Treinta especies de este polígono son raras, pues solamente se conocen en una celda dentro de su territorio. Una posible ruta de conectividad entre los polígonos que constituyen la RAC se muestra en la figura $3 \mathrm{~B}$, dicha ruta liga los centroides geográficos de cada polígono por su ruta más corta, minimizando a su vez la superficie adicional que se requiere para establecer una posible conectividad entre todos los polígonos seleccionados como importantes para la conservación de las especies.

La cobertura vegetal de la Sierra Norte abarca $6949.4 \mathrm{~km}^{2}$ (77.8\% de su territorio), mientras que 1976.3 $\mathrm{km}^{2}(22.1 \%)$ han sido transformados en zonas urbanas, agrícolas o ganaderas (Cuadro 5). En la Sierra se encuen-
Cuadro 4: Prioridad de selección de los principales polígonos que constituyen la red de áreas prioritarias de conservación. Los polígonos fueron definidos utilizando la riqueza de especies de Asteraceae registradas en la Red de Áreas de Conservación (RAC) definida por el algoritmo MDS2 del programa ConsNet en la Sierra Norte de Oaxaca, México.

\begin{tabular}{ccccc}
\hline Prioridad & Polígono & \multicolumn{2}{c}{ Riqueza } & Área $\left(\mathrm{km}^{2}\right)$ \\
\cline { 3 - 4 } & & $\mathrm{N}=225$ & Complemento & \\
\hline 1 & Ixtlán 1 & $144(64 \%)$ & 81 & 490.58 \\
2 & Mixe 1 & $54(88 \%)$ & 48 & 85.38 \\
3 & Ixtlán 2 & $15(94.6 \%)$ & 13 & 41.39 \\
4 & Villa Alta- & $4(96.4 \%)$ & 4 & 71.05 \\
5 & Mixe & & & \\
6 & Ixtlán 3 & $3(97.7 \%)$ & 3 & 15.98 \\
7 & Mixe 2 & $2(98.6 \%)$ & 2 & 15.13 \\
8 & Ixtlán 4 & $2(99.5 \%)$ & 2 & 1.26 \\
& Villa Alta & $1(100 \%)$ & 1 & 9.38 \\
\hline
\end{tabular}

Cuadro 5: Número de especies que presentan los tipos de vegetación registrados en la Sierra Norte de Oaxaca, México (adaptado de INEGI, 2015).

\begin{tabular}{|c|c|c|c|c|}
\hline Tipo de vegetación & Superficie $\left(\mathrm{km}^{2}\right)$ & $\%$ Sierra Norte & Especies & Registros \\
\hline Bosque húmedo de montaña & 776.61 & 8.70 & 74 & 145 \\
\hline Bosque tropical caducifolio & 1.36 & 0.01 & 0 & 0 \\
\hline Bosque tropical perennifolio & 555.35 & 6.22 & 29 & 65 \\
\hline Bosque de Pinus & 130.29 & 1.46 & 26 & 64 \\
\hline Bosque de Quercus & 150.48 & 1.68 & 12 & 14 \\
\hline Bosque de oyamel (Abies) & 6.82 & 0.08 & 2 & 2 \\
\hline Vegetación secundaria derivada de bosque húmedo de montaña & 1324.34 & 14.84 & 129 & 469 \\
\hline Vegetación secundaria derivada de bosque tropical caducifolio & 157.95 & 1.77 & 5 & 7 \\
\hline Vegetación secundaria derivada de bosque de Quercus & 338.87 & 3.79 & 19 & 23 \\
\hline Zonas agrícolas & 937.58 & 10.53 & 142 & 271 \\
\hline Pastizal inducido & 915.29 & 10.25 & 2 & 2 \\
\hline Plantaciones forestales & 113.23 & 1.27 & 0 & 0 \\
\hline \multirow[t]{2}{*}{ Zonas urbanas } & 10.17 & 0.11 & 2 & 2 \\
\hline & 8925.7 & 100 & $\mathrm{~N}=342$ & $\mathrm{~N}=1560$ \\
\hline
\end{tabular}


tran siete tipos de vegetación y comunidades secundarias derivadas de ellos (Cuadro 5). Estos tipos de vegetación, agrupados por biomas indican que los bosques templados $\mathrm{y}$ el bosque húmedo de montaña son los de mayor cobertura y los que también albergan el mayor número de especies analizadas.

El Cuadro 6 muestra el número de especies incluidas en la RAC distribuidas por bioma. La mayor riqueza se observa en el bosque húmedo de montaña y el bosque tropical húmedo; ambos biomas también ocupan gran parte del territorio de la Sierra Norte. Otro conjunto importante de especies se reporta de las zonas agrícolas, indicando el papel relevante de la familia por su componente de especies arvenses y ruderales.

\section{Discusión}

La Sierra Norte de Oaxaca comprende una importante riqueza representativa de la flora del estado, la más diversa del país. La región contiene $26.4 \%$ de toda la riqueza conocida del estado, incluyendo un importante número de especies endémicas de México, de Oaxaca y endemismos locales conocidos solamente de partes de la Sierra (Cuadro 1). La relevancia de la región ya había sido detectada previamente; por ejemplo, la Comisión Nacional para el Conocimiento y Uso de la Biodiversidad (CONABIO) la consideró dentro de las zonas prioritarias para la conser- vación de la biodiversidad (Arriaga et al., 2000), constituyendo la región prioritaria 130 (Sierra del Norte de Oaxaca-Mixe). Desafortunadamente, aunque ya fue identificada desde hace más de tres lustros como región prioritaria para la conservación, hasta la fecha no hay acciones encaminadas a proponer zonas de protección de su flora y fauna. En la Sierra se identifican 87 especies endémicas locales, 11 de ellas miembros de la familia Asteraceae evaluadas en este trabajo; todas ellas requieren acciones específicas de conservación, dado que ocupan sitios considerados como irremplazables para su conservación a largo plazo.

Las especies de Asteraceae prefieren sitios con una alta heterogeneidad ambiental, como los ocasionados por los abruptos cambios de altitud. En la Sierra Norte se encuentran características fisiográficas cambiantes en distancias cortas, presencia de arroyos, así como cambios en la pendiente y la exposición de las laderas. Tal heterogeneidad ambiental favorece la presencia de un importante número de especies de Asteraceae, con una preferencia por altitudes medias (2000-2400 m). Los resultados apoyan lo discutido por Boyle (1996), quién encontró en la Sierra Norte una disminución en el número de familias, géneros y especies con los cambios de altitud. Ya antes Nix (1986) afirmaba también que a mayor variabilidad ambiental, mayor variabilidad biológica.

Cuadro 6: Área que ocupan los principales biomas distribuidos en la Sierra Norte de Oaxaca y el total de especies que registran. Las dos últimas columnas muestran el número de especies (Spp RAC) y de registros (Registros (RAC)) incluidos en la red de áreas de conservación (RAC), correspondientes a cada bioma. BTES=Bosque tropical estacionalmente seco, BHM=Bosque húmedo de montaña, BTEM=Bosque templado, $\mathrm{BTH}=$ Bosque tropical húmedo.

\begin{tabular}{|c|c|c|c|c|c|c|}
\hline Bioma & Área $\left(\mathrm{km}^{2}\right)$ & $\%$ de la Sierra Norte & Total de Especies & Total de Registros & Spp. RAC & Registros RAC \\
\hline Agricultura & 1060.97 & 11.89 & 109 & 273 & 46 & 60 \\
\hline BTES & 159.31 & 1.79 & 5 & 7 & 2 & 2 \\
\hline BHM & 2100.95 & 23.54 & 117 & 614 & 111 & 456 \\
\hline BTEM & 1235.97 & 13.85 & 36 & 76 & 11 & 17 \\
\hline BTH & 3453.15 & 38.69 & 117 & 588 & 84 & 204 \\
\hline Sin vegetación & 915.29 & 10.25 & 2 & 2 & 1 & 1 \\
\hline Total & 8925.67 & 100 & --- & 1560 & --- & 740 \\
\hline
\end{tabular}


La mayoría de las especies de Asteraceae analizadas se encuentran con frecuencia en vegetación secundaria, principalmente la derivada del bosque húmedo de montaña o de los bosques templados, los biomas mejor representados en la región de estudio (Cuadro 6). Muchas especies de Asteraceae son heliófilas favorecidas por el aclareo de la vegetación original, aunque otros miembros forman parte de la vegetación primaria. Muchas de sus especies se encuentran amenazadas por las actividades humanas, sobre todo por el aprovechamiento forestal extendido por toda la Sierra. Por lo tanto, es importante considerar el impacto que la actividad forestal tiene en las estrategias futuras de conservación, pues constituye la base económica de la mayoría de las comunidades rurales.

Aunque no existen áreas de conservación con decreto federal o estatal en la Sierra Norte, las comunidades han planteado el establecimiento de "Zonas de conservación indígena-comunitarias" (ICCAs) (Robson, 2007; Martin et al., 2011) como parte de sus programas de manejo forestal. Desafortunadamente, en estas zonas no quedan incluidas áreas de distribución de las especies endémicas analizadas. La red de áreas de conservación obtenida (Fig. 3A) incluye algunas de estas zonas (ICCAs), las cuales fueron corroboradas durante el trabajo de campo. Sin embargo, aún no se cuenta con cartografía que permita conocer con precisión los límites geográficos de tales ICCAs.

El modelado de la distribución potencial de la biodiversidad se ha desarrollado como una línea de investigación ecológica importante durante las últimas dos décadas (Ferrier y Watson, 1997; Guisan y Zimmermann, 2000; Peterson, 2001; Villaseñor y Téllez-Valdés, 2004; Mouquet et al., 2015). Los Modelos de Nicho Ecológico (MNE) de las especies también han sido provechosos para conocer cuáles son los factores ambientales que determinan la distribución de la biodiversidad a través de diferentes escalas. Además, sirven como pronósticos de la biodiversidad a las presiones ambientales, cambio en el uso del suelo, invasión de especies no nativas y las interacciones entre especies (Ficetola et al., 2010; Vicente et al., 2011; Petitpierre et al., 2012; Guisan et al., 2013;
Gonçalves et al., 2016). Todos estos análisis realizados con base en MNE han sido útiles para complementar información sobre la estructura, manejo y conservación de ecosistemas, como es el caso de la Sierra Norte. Los MNE obtenidos en este estudio han permitido reconocer la distribución potencial de las especies endémicas empleadas como substitutos de la biodiversidad; con ello se reconocen áreas prioritarias de conservación (Fig. 3). Es necesario, sin embargo, hacer esfuerzos de trabajo de campo para corroborar valores de riqueza en sitios importantes detectados o para identificar sitios con riqueza florística importante de esta RAC.

El ritmo de deforestación en muchas regiones de Oaxaca requiere de planes urgentes para conservar su biodiversidad, su funcionamiento ecológico y la provisión de bienes y servicios ambientales que mantienen. Sin embargo, la estimación directa de la biodiversidad en estas zonas presenta dificultades metodológicas, especialmente por la carencia de datos de calidad sobre la distribución de las especies, la accesibilidad limitada y la escasez de medios técnicos y económicos para llevar a cabo estudios de campo. Un ejemplo específico es el distrito Mixe, donde la información florística es más escasa que en los distritos de Ixtlán y Villa Alta. El distrito es difícil de acceder por lo complejo del terreno, clima y, por ser una de las zonas de la Sierra Norte con mayores conflictos sociales, que fomentan altos niveles de inseguridad por la falta de acercamiento con los líderes comunitarios, quienes además rechazan autorizar los estudios florísticos. Por ello los MNE, como se ilustra en este ejercicio, pueden ser una herramienta de gran utilidad en el diseño de estrategias de conservación en zonas donde la información es aún insuficiente.

La RAC obtenida con los MNE permitiría proteger una importante cantidad de especies (256), 30 de ellas conocidas de un solo un registro. El polígono Ixtlán 1 concentra la mayor cantidad de especies, lo que lo ubica como la región con mayor relevancia para el establecimiento de algún tipo de reserva; sin embargo, no hay que soslayar que también cuenta con mayor esfuerzo de recolecta (Apéndice). Por otro lado hay que considerar que Mixe 1 constituye otro polígono con alta concentración 
de endemismos, ubicado en el distrito con menor número de recolectas. Si fuera posible ampliar el conocimiento de la diversidad y distribución del endemismo en este distrito, su relevancia se ponderaría mejor y se tendría una estimación más precisa de su riqueza biológica (Cuadro 4).

En los resultados obtenidos se identifican ocho áreas importantes donde se podrían plantear estrategias de conservación en la Sierra Norte de Oaxaca. El uso de especies de Asteraceae como subrogados de la biodiversidad (Villaseñor et al., 2007; Suárez-Mota et al., 2015; 2017) funcionaría como eje para concentrar futuros esfuerzos de inventario y de protección de la riqueza florística en tales sitios. La Red de Áreas de Conservación (RAC) estructurada en este trabajo permitirá en un futuro detectar y seleccionar, con bases conceptuales más sólidas, áreas que se integren al SINAP y a mitigar los efectos de la degradación de la cobertura vegetal tan pronunciada en la región bajo estudio. La RAC mostrada en la figura $3 \mathrm{~A}$ toma en cuenta los sitios irremplazables, es decir, aquellos que registran especies conocidas solamente en esa porción de territorio. Desafortunadamente la RAC no considera otras 63 celdas identificadas como irremplazables. Además, incluye poco más de la mitad de las especies nativas y/o endémicas de México registradas en la zona de estudio, así como importantes zonas con vegetación primaria, aún no afectadas por las actividades humanas (Cuadro 6).

\section{CONTRIBUCIÓN DE AUTORES}

MESM realizó los modelos de nicho ecológico y los análisis de complementariedad, JLV contribuyó sustancialmente en los análisis y el manuscrito mientras que MBRA estructuró la base de datos obtenida con datos de campo y realizo también modelos de nicho ecológico con la asesoría de MESM. Todos los autores contribuyeron a la discusión, revisión y aprobación del manuscrito final.

\section{FINANCIAMIENTO}

Este estudio fue apoyado por la Secretaría de Educación Pública (SEP, Proyecto PRODEP-UNSIJ-PTC-028) otorgado al primer autor.

\section{AgradeCIMIENTOS}

Agradecemos a Irene Bautista Juárez, Teresa Martínez Martínez, Diana Nava Juárez y Ángel Mario Muñoz Juárez su apoyo en el trabajo de recolecta y acopio de datos en el campo, así como por su participación en la actualización de la base de datos. Se agradece también la contribución de Enrique Ortiz en el manejo de la información en la base de datos. De igual manera agradecemos a los editores y revisores anónimos por sus valiosas sugerencias.

\section{LITERATURA CITADA}

Araújo, M. B. y P. H. Williams. 2000. Selecting areas for species persistence using ocurrence data. Biological Conservation 96(3): 331-345. DOI: https://doi.org/10.1016/s00063207(00)00074-4

Araújo, M. B., W. Thuiller, R. G. Pearson. 2006. Climate warming and the decline of amphibians and reptiles in Europe. Journal of Biogeography 34(1): 1712-1728. DOI: https://dx.doi.org/10.1111/j.1365-2699.2006.01482.x

Arriaga, L., J. M. Espinoza, C. Aguilar, E. Martínez, L. Gómez y E. Loa. (coord.). 2000. Regiones terrestres prioritarias de México. Comisión Nacional para el Conocimiento y uso de la Biodiversidad. México, D.F., México. 810 pp.

Austin, M. P. 1998. An ecological perspective on biodiversity investigations: examples from Australian eucalypt forests. Annals of the Missouri Botanical Garden 85: 2-17.

Austin, M. P. 2002. Case studies of the use of environmental gradients in vegetation and fauna modelling: theory and practice in Australia and New Zealand. In: Scott, J. M., P. J. Heglund, M. Morrison, J. Haufler, M. Raphael, B. Wall y F. Samson (eds.). Predicting Species Occurrences: Issues of Accuracy and Scale. Island Press. Covelo, USA. pp. 73-82.

Balleza, J. J. y J. L. Villaseñor. 2002. La familia Asteraceae en el estado de Zacatecas (México). Acta Botanica Mexicana 59: 5-69. DOI: http://dx.doi.org/1021829/ abm59.2002.893

Boyle, B. L. 1996. Changes in altitudinal and latitudinal gradients. Tesis doctoral. School of Arts and Sciences, Washington University. Washington, D.C., USA. pp. 275. 
Busby, J. R. 1986. A biogeoclimatic analysis of Nothofagus cunninghamii (Hook) Oerst. in southern Australia. Australian Journal of Ecology 11(1): 1-7.

Cain, S. A. 1950. Life forms and phytoclimates. The Botanical Review 16(1): 1-32. DOI: https://doi.org/10.1007/ BF02879783

Castellanos-Bolaños, J. F., E. J. Treviño-Garza, O. A. AguirreCalderón, J. Jiménez-Pérez, y A. Velázquez-Martínez. 2010. Diversidad arbórea y estructura espacial de bosques de pino-encino en Ixtlán de Juárez, Oaxaca. Revista Mexicana de Ciencias Forestales 1(2): 39-52.

Ceballos, G., P. R. Ehrlich, J. Soberón, I. Salazar y J. P. Fay. 2005. Global mammal conservation: what must we manage? Science 309(5734): 603-607. DOI: https://doi. org/10.1126/science. 1114015

Chapman, A. D. y J. R. Busby. 1994. Linking plant species information to continental biodiversity inventory, climate modeling and environmental monitoring. Mapping the diversity of nature. Chapman \& Hall. London, UK. pp. 179-195.

Ciarleglio, M., S. Sarkar y J. W. Barnes. 2008. ConsNet manual: V.1.10. Biodiversity and Biocultural Conservation Laboratory, University of Texas at Austin. Austin, USA. $47 \mathrm{pp}$.

Ciarleglio, M., J. W. Barnes y S. Sarkar. 2009. ConsNet: new software for the selection of CANs with spatial and multicriteria analyses. Ecography 32(2): 205-209. DOI: https:// doi.org/10.1111/j.1600-0587.2008.05721.x

Cox, C. B., I. N. Healey y P. D. Moore. 1976. Biogeography: an ecological and evolutionary approach. Blackwell Scientific Publications. Oxford, USA. 244 pp.

Cuervo-Robayo, A., O. Téllez-Valdés, M. A. Gómez-Albores, C. S. Venegas-Barrera, J. Manjarrez y E. Martínez-Meyer. 2013. An update of high-resolution monthly climate surface for Mexico. International Journal of Climatology 34(7): 2427-2437. DOI: https://doi.org/10.1002/joc.3848

Elith, J. y M. A. Burgman. 2002. Habitat models for Popultion Viability Analysis. In: Brigham, C. A., M. W. Schwanz (eds.). Population Viability in Plants. Springer. Berlin, Germany. pp. 203-235. DOI: https://doi.org/10.1007/9783-662-09389-4
EMCV. 2008. Estrategia Mexicana para la Conservación Vegetal: Objetivos y Metas. Comisión Nacional para el Conocimiento y Uso de la Biodiversidad (CONABIO), Comisión Nacional de Áreas Naturales Protegidas (CONANP), Secretaría de Medio Ambiente y Recursos Naturales (SEMARNAT). México, D.F., México. 36 pp.

Engler, R., C. F. Randin, P. Vittoz, T. Czáka, M. Beniston, N. E. Zimmermann y G. Guisan. 2009. Predicting future distributions of mountain plants under climate change: does dispersal capacity matter? Ecography 32(1): 34-45. DOI: https://doi.org/10.1111/j.1600-0587.2009.05789.x

ESRI. 2011. Maps throughout this article were created using Arc-GIS ${ }^{\circ}$ software. Environmental Systems Research Institute (ESRI). Redlands, USA.

Ferrier, S. y G. Watson. 1997. An evaluation of the effectiveness of environmental surrogates and modelling techniques in predicting the distribution of biological diversity. Department of the Environment, Australian Government. Canberra, Australia. 184 pp.

Ficetola, G. F., L. Maiorano, A. Falcucci, N. Dendoncker, L. Boitani, E. Padoa-Schioppa, C. Miaud y W. Thuiller. 2010. Knowing the past to predict the future: landuse change and the distribution of invasive bullfrogs. Global Change Biology 16(2): 528-537. DOI: https://doi. org/10.1111/j.1365-2486.2009.01957.x

Fischer, J., D. B. Lindenmayer, H. A. Nix, J. L. Stein y J. A. Stein. 2001. Climate and animal distribution: a climatic analysis of the Australian marsupial Trichosurus caninus. Journal of Biogeography 28(3): 293-304. DOI: https:// doi.org/10.1046/j.1365-2699.2001.00554.X

Franklin, J. 1995. Predictive vegetation mapping: geographic modelling of biospatial patterns in relation to environmental gradients. Progress in Physical Geography 19(4): 474499. DOI: https://doi.org/10.1177/030913339501900403

García-Mendoza, A. J. 2004. Integración del conocimiento florístico del Estado. In: García-Mendoza, A. J., M. J. Ordoñez y M. Briones-Salas (eds.). Biodiversidad de Oaxaca. Instituto de Biología de la Universidad Nacional Autónoma de México (UNAM), Fondo Oaxaqueño para la Conservación de la Naturaleza, World Wildlife Found (WWF). México, D.F., México. pp. 305-325. 
GBIF. 2017. Global Biodiversity Information Facility. http:// www.gbif.org/ (consultado abril de 2017).

Gonçalves, J., J. P. Honrado, J. R. Vicente y E. Civantos. 2016. A model-based framework for assessing the vulnerability of low dispersal vertebrates to landscape fragmentation under environmental change. Ecological Complexity 28: 174-186. DOI: https://doi.org/10.1016/j. ecocom.2016.05.003

Guisan, A. y N. E. Zimmermann. 2000. Predictive habitat distribution models in ecology. Ecological Modelling 135(2-3): 147-186. DOI: https://doi.org/10.1016/s03043800(00)00354-9

Guisan, A., R. Tingley, J. B. Baumgartner, I. Naujokaitis-Lewis, P. R. Sutcliffe, A. I. T. Tulloch, J. T. Regan, L. Brotons, E. McDonald-Maddem, C. Mantyka-Pringle, G. T. Martin, R. J. Rhodes, R. Maggini, A. S. Stterfield, J. Elith, W. M. Schwartz, A. B. Wintle, O. Broennimann, M. Austin, S. Ferrier, R. Kearney, P. H. Possingham y M. Y. Buckley. 2013. Predicting species distributions for conservation decisions. Ecology Letters 16(12): 1424-1435. DOI: https://doi.org/10.1111/ele.12189

Holdridge, L. R. 1967. Life zone ecology. Tropical Science Center. Saint Joseph, Costa Rica. 206 pp.

Hutchinson, G. E. 1957. Concluding remarks. Cold Spring Harbor Symposia on Quantitative Biology 22: 415-427. DOI: https://doi.org/10.1101/sqb.1957.022.01.039

INEGI. 2004a. Información de Climas escala 1: 1,000,000. Instituto Nacional de Estadística Geografía e Informática. Aguascalientes, México.

INEGI. 2004b. Continuo nacional de datos alfanumérico edafológico escala 1: 250,000 Serie I. Instituto Nacional de Estadística Geografía e Informática. Aguascalientes, México.

INEGI. 2005. Conjunto de datos vectoriales geológicos, continuo nacional escala 1: 1,000,000. Instituto Nacional de Estadística Geografía e Informática. Aguascalientes, México.

INEGI. 2015. Conjunto de datos vectoriales de uso de suelo y vegetación escala 1:250,000. Serie V. Instituto Nacional de Estadística Geografía e Informática. Aguascalientes, México.
Koleff, P., A. Lira-Noriega, C. Cantú, T. Urquiza y M. Kolb. 2007. Análisis de vacíos y omisiones en conservación de la biodiversidad terrestre de México: espacios y especies. Comisión Nacional para el Conocimiento y Uso de la Biodiversidad (CONABIO), Comisión Nacional de Áreas Naturales Protegidas (CONANP), The Nature Conservancy-Programa México (TNC), Pronatura, A.C. (PRONATURA), Secretaría de Medio Ambiente y Recursos Naturales (SEMARNAT), Convenio Sobre la Biodiversidad Biológica (CDB), Universidad Autónoma de Nuevo León (UANL). México, D.F., México. 128 pp.

Lindenmayer, D. B., B. G. Mackey y H. A. Nix. 1996. The bioclimatic domains of four species of commercially important eucalypts from south-eastern Australia. Australian Forestry 59(2): 74-89. DOI: https://doi.org/10. 1080/00049158.1996.10674672

Lorence, D. H. y A. García-Mendoza. 1989. Oaxaca, México. In: Campbell, D. G. y H. D. Hammond (eds.). Floristic inventory of tropical countries: the status of plant systematics, collections, and vegetation, plus recommendations for the future. New York Botanical Garden. New York, USA. pp. 253-269.

Margules, C. y S. Sarkar. 2009. Planeación Sistemática de la Conservación. Universidad Nacional Autónoma de México (UNAM), Comisión Nacional de Áreas Naturales Protegidas (CONANP), Comisión Nacional para el Conocimiento y Uso de la Biodiversidad (CONABIO). México, D.F., México. 279 pp.

Martin, G. J., C. I. B. Camacho, C. A. G del Campo, S. A. Fonseca, F. C. Mendoza y M. A. G. Ortiz. 2011. Indigenous and community conserved areas in Oaxaca, Mexico. Management of Environmental Quality 22(2): 250-266. DOI: https://doi.org/10.1108/14777831111113419

Mouquet, N., Y. Lagadeuc, V. Devictor, L. Doyen, A. Duputié, D. Eveillard, D. Faure, E. Garnier, O. Gimenez, P. Huneman, F. Jabot, P. Jarne, D. Joly, R. Julliard, S. Kéfi, J. G. Kergoat, S. Lavorel, L. L. Gall, L. Meslin, S. Morand, X. Morin, H. Morlon, G. Pinay, R. Pradel, M. F. Schurr, W. Thuiller y M. Loreau. 2015. Predictive ecology in a changing world. Journal of Applied Ecology 52: 12931310. DOI: https://doi.org/10.1111/1365-2664.12482 
Myers, N., R. A. Mittermeier, C. G. Mittermeier, G. A. B. da Fonseca y J. Kent. 2000. Biodiversity hotspots for conservation priorities. Nature 403(6772): 853-858. DOI: https://doi.org/10.1038/35002501

Narro, R. J. 2010. Flora y fauna mexicanas de los centenarios. Instituto de Biología Universidad, Nacional Autónoma de México. México, D.F., México. pp. 129.

Nix, H. A. 1986. A biogeographic analysis of elapid snakes. In: Longmore, E. (ed.). Atlas of Elapid snakes of Australia, Australia Flora and fauna series. Australian Government Publishing Service. Canberra, Australia. pp. 4-15.

Palacio-Prieto, J. L., G. Bocco, A. Velázquez, J. F. Mas, F. Takaki-Takaki, A. Victoria, L. Luna-González, G. Gómez-Rodríguez, J. López-García, M. Palma, I. TrejoVázquez, A. Peralta, J. Prado-Molina, A. RodríguezAguilar, R. Mayorga-Saucedo y F. González Medrano. 2000. La condición actual de los recursos forestales en México: Resultados del Inventario Forestal Nacional 2000. Investigaciones geográficas 43: 183-203.

Peterson, A. T. 2001. Predicting species geographic distributions based on ecological niche modeling. Condor 103(3): 599-605. DOI: https://doi.org/10.1650/00105422(2001)103\%5B0599:PSGDBO\%5D2.0.CO;2

Peterson, A. T., L. G. Ball y K. P. Cohoon. 2002. Predicting distributions of Mexican birds using ecological niche modelling methods. Ibis 144(1): 27-32. DOI: https://doi. org/10.1046/j.0019-1019.2001.00031.x

Petitpierre, B., C. Kueffer, O. Broennimann, C. Randin, C. Daehler y A. Guisan. 2012. Climatic niche shifts are rare among terrestrial plant invaders. Science 335(6074): 13441348. DOI: https://doi.org/10.1126/science.1215933

Phillips, S. J. y M. Dudík. 2008. Modeling of species distributions with MaxEnt: new extensions and a comprehensive evaluation. Ecography 31(2): 161-175. DOI: https://doi. org/10.1111/j.0906-7590.2008.5203.x

Real, R., A. M. Barbosa, A. Rodríguez, F. J. García, J. M. Vargas, L. J. Palomo y M. Delibes. 2009. Conservation biogeography of ecologically interacting species: the case of the Iberian lynx and the European rabbit. Diversity and Distributions 15(3): 390-400. DOI: https://doi. org/10.1111/j.1472-4642.2008.00546.x
REMIB. 2017. Especies de la Familia Asteraceae. Red Mundial de Información sobre Biodiversidad. http://www.conabio. gob.mx/remib. (consultado marzo de 2017).

Ricker, M., I. Ramírez-Krauss, G. Ibarra-Manríquez, E. Martínez, C. Ramo, G. González-Medellín, G. GómezRodríguez, J. L. Palacio-Prieto y H. M. Hernández. 2007. Optimizing conservation of forest diversity: a countrywide approach in Mexico. Biodiversity and Conservation 16(6): 1927-1957. DOI: https://doi.org/10.1007/s10531006-9112-z

Robson, J. P. 2007. Local approaches to biodiversity conservation: Lesson from Oaxaca, southern Mexico. International Journal of Sustainable Development 10(3): 267-286. DOI: https://doi.org/10.1504/ijsd.2007.017647

Rzedowski, J. 1998. Diversidad y orígenes de la flora fanerogámica de México. Diversidad biológica de México: orígenes y su distribución. In: Ramamoorthy, $\mathrm{T}$. P., R. Bye, A. Lot y J. Fa (comps.). Instituto de Biología, Universidad Nacional Autónoma de México. México, D.F., México. pp. 129-145.

Rzedowski, J. y T. Reyna-Trujillo. 1990. Vegetación potencial Mapa IV.8.2. Atlas Nacional de México Instituto de Geografía, Universidad Nacional Autónoma de México. México, D.F., México.

Scott, J. M., P. J. Heglund, F. Samson, J. Haufler, M. Morrison, M. Raphael y B. Wall. 2002. Predicting Species Occurrences: Issues of Accuracy and Scale. Island Press. Covelo, USA. 868 pp.

Shugart, H. H. 1990. Using ecosystem models to assess potential consequences of global climatic change. Trends in Ecology and Evolution 5(9): 303-307. DOI: https://doi. org/10.1016/0169-5347(90)90086-s

SINAP. 2014. Áreas Protegidas del Estado de Oaxaca. Sistema Nacional de Áreas Protegidas. https://simec.conanp.gob. $\mathrm{mx} /$ Info (consultado noviembre de 2016).

Soberón, J. y A. T. Peterson. 2005. Interpretation of models of fundamental ecological niches and species' distributional areas. Biodiversity Informatics 2: 1-10. DOI: https://doi. org/10.17161/bi.v2i0.4

Suárez-Mota, M. E. y J. L. Villaseñor. 2011. Las compuestas endémicas de Oaxaca, México: Diversidad y Distribución. 
Boletín de la Sociedad Botánica de México 88: 55-66. DOI: https://doi.org/10.17129/botsci.308

Suárez-Mota, M. E. y O. Téllez-Valdés. 2014. Red de áreas prioritarias para la conservación de la biodiversidad del eje volcánico transmexicano analizando su riqueza florística y variabilidad climática. Polibotánica 38: 67-93.

Suárez-Mota, M. E., J. L. Villaseñor y L. López-Mata. 2015. La región del Bajío, México y la Conservación de su diversidad florística. Revista Mexicana de Biodiversidad 86(3): 799-808. DOI: https://doi.org/10.1016/j. rmb.2015.06.001

Suárez-Mota, M. E., J. L. Villaseñor y L. López-Mata. 2017. Dominios climáticos de la Sierra Madre Oriental y su relación con la diversidad florística. Revista Mexicana de Biodiversidad 88(1): 224-233. DOI: https://dx.doi. org/10.1016/j.rmb.2017.01.020

Sykes, M. T., I. C. Prentice y W. Cramer. 1996. A bioclimatic model for the potential distribution of north European tree species under present and future climates. Journal of Biogeography 23(2): 203-233.

Teixeira, J. y J. W. Arntzen. 2002. Potential impact of climate warming on the distribution of the Golden-striped salamander, Chioglossa lusitanica, on the Iberian Peninsula. Biodiversity and Conservation 11(12): 21672176.

Torres-Colín, R., H. D. Lorence, de A. M. P. Ramírez y A. R. E. Villa. 2009. Flora de la Sierra de Juárez, Oaxaca: Distrito de Ixtlán y áreas adyacentes (Sierra Norte de Oaxaca). Listados Florísticos de México XXV: 7-79.

UNIBIO. 2017. Ejemplares de la Familia Asteraceae Unidad de Informática para la Biodiversidad. http://unibio.unam. mx. (consultado febrero de 2017).

Vásquez-Cortés, V. 2013. Estructura, composición y diversidad arbórea en áreas de manejo forestal de Ixtlán de Juárez, Oaxaca. Tesis de licenciatura. Universidad de la Sierra Juárez. Oaxaca, México. 30 pp.
Vicente, J., C. F. Randin, J. Gonçalves, M. J. Metzger, A. Lomba, J. Honrado y A. Guisan. 2011. Where will conflicts between alien and rare species occur after climate and land-use change? A test with a novel combined modelling approach. Biological Invasions 13(5): 1209-1227. DOI: https://doi.org/10.1007/s10530-011-9952-7

Villaseñor, J. L. 1993. La familia Compositae en México. Revista de la Sociedad Mexicana de Historia Natural 44: 117-124.

Villaseñor, J. L. 2004. Los géneros de plantas vasculares de la flora de México. Boletín de la Sociedad Botánica de México 75: 105-135.

Villaseñor, J. L. 2016. Checklist of the native vascular plants of Mexico. Revista Mexicana de Biodiversidad 87(3): 559902. DOI: https://doi.org/10.1016/j.rmb.2016.06.017

Villaseñor, J. L. y O. Téllez-Valdés. 2004. Distribución potencial de las especies del género Jefea (Asteraceae) en México. Anales del Instituto de Biología, Universidad Nacional Autónoma de México, Serie Botánica 75: 205-220.

Villaseñor, J. L., G. Ibarra-Manríquez, J. A. Meave y E. Ortiz. 2005. Higher taxa as surrogates of plant biodiversity in a megadiverse country. Conservation Biology 19(1): 232-238. DOI: https://doi.org/10.1111/j.15231739.2005.00264.x

Villaseñor, J. L., P. Maeda, J. A. Rosell y E. Ortiz. 2007. Plant families as predictors of plant biodiversity in Mexico. Diversity and Distributions 13(6): 871-876. DOI: https:// doi.org/10.1111/j.1472-4642.2007.00385.x

Villaseñor, J. L. y E. Ortiz. 2012. La familia Asteraceae en la Flora del Bajío y de regiones adyacentes. Acta Botanica Mexicana 100: 263-295. DOI: http://dx.doi.org/10.21829/ abm100.2012.37

Villaseñor, J. L. y E. Ortiz. 2014. Biodiversidad de las plantas con flores (División Magnoliophyta) en México. Revista Mexicana de Biodiversidad 85(S): 134-142. DOI: https:// dx.doi.org/10.7550/rmb.31987. 
Apéndice. Especies de Asteraceae registradas en la Sierra Norte de Oaxaca, México. Se indica el número de registros provenientes de ejemplares de herbario identificados y georreferenciados. Su presencia en los distritos Ixtlán, Mixe y Villa Alta se indica con un 1.

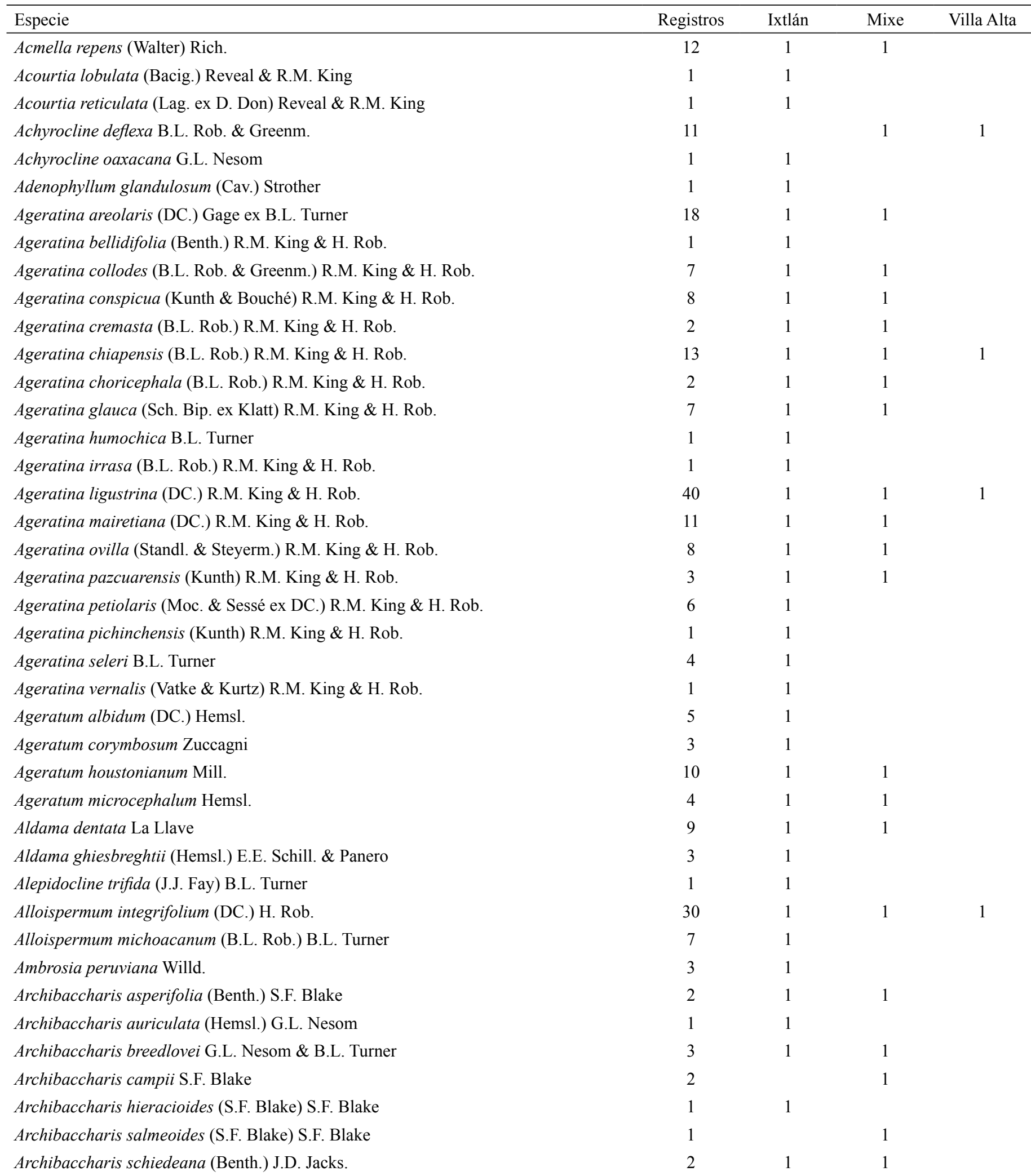


Apéndice. Continuación.

\begin{tabular}{|c|c|c|c|c|}
\hline Especie & Registros & Ixtlán & Mixe & Villa Alta \\
\hline Archibaccharis serratifolia (Kunth) S.F. Blake & 1 & & 1 & \\
\hline Archibaccharis trichotoma (Klatt) G.L. Nesom & 10 & 1 & 1 & \\
\hline Aztecaster pyramidatus (B.L. Rob. \& Greenm.) G.L. Nesom & 1 & 1 & & \\
\hline Baccharis conferta Kunth & 6 & 1 & 1 & \\
\hline Baccharis mexicana Cuatrec. & 2 & 1 & & \\
\hline Baccharis multiflora Kunth & 17 & 1 & 1 & \\
\hline Baccharis pteronioides DC. & 1 & 1 & & \\
\hline Baccharis salicifolia (Ruiz \& Pav.) Pers. & 11 & 1 & 1 & \\
\hline Bartlettina calderonii (B.L. Turner) B.L. Turner & 3 & 1 & & \\
\hline Bartlettina constipatiflora (Klatt) R.M. King \& H. Rob. & 8 & 1 & 1 & \\
\hline Bartlettina karwinskiana (DC.) R.M. King \& H. Rob. & 12 & 1 & 1 & \\
\hline Bartlettina macdougallii R.M. King \& H. Rob. & 13 & 1 & 1 & \\
\hline Bartlettina oresbia (B.L. Rob.) R.M. King \& H. Rob. & 6 & & 1 & \\
\hline Bartlettina platyphylla (B.L. Rob.) R.M. King \& H. Rob. & 2 & 1 & & \\
\hline Bartlettina sordida (Less.) R.M. King \& H. Rob. & 25 & 1 & 1 & \\
\hline Bartlettina tuerckheimii (Klatt) R.M. King \& H. Rob. & 13 & 1 & 1 & \\
\hline Bidens alba (L.) DC. & 1 & 1 & & \\
\hline Bidens sharpii (Sherff) Melchert & 3 & 1 & & \\
\hline Bidens squarrosa Kunth & 7 & 1 & 1 & \\
\hline Bidens triplinervia Kunth & 16 & 1 & 1 & 1 \\
\hline Brickellia argyrolepis B.L. Rob. & 2 & 1 & & \\
\hline Brickellia glandulosa (La Llave) McVaugh & 3 & 1 & & \\
\hline Brickellia veronicifolia (Kunth) A. Gray & 1 & 1 & & \\
\hline Calea ternifolia Kunth & 14 & 1 & 1 & 1 \\
\hline Calea urticifolia (Mill.) DC. & 3 & & 1 & 1 \\
\hline Calendula officinalis L. & 4 & 1 & & \\
\hline Cirsium anartiolepis Petr. & 1 & 1 & & \\
\hline Cirsium ehrenbergii Sch. Bip. & 1 & 1 & & \\
\hline Cirsium lappoides (Less.) Sch. Bip. & 1 & & & 1 \\
\hline Cirsium mexicanum DC. & 6 & 1 & 1 & \\
\hline Cirsium pinetorum Greenm. & 1 & 1 & & \\
\hline
\end{tabular}


Apéndice. Continuación.

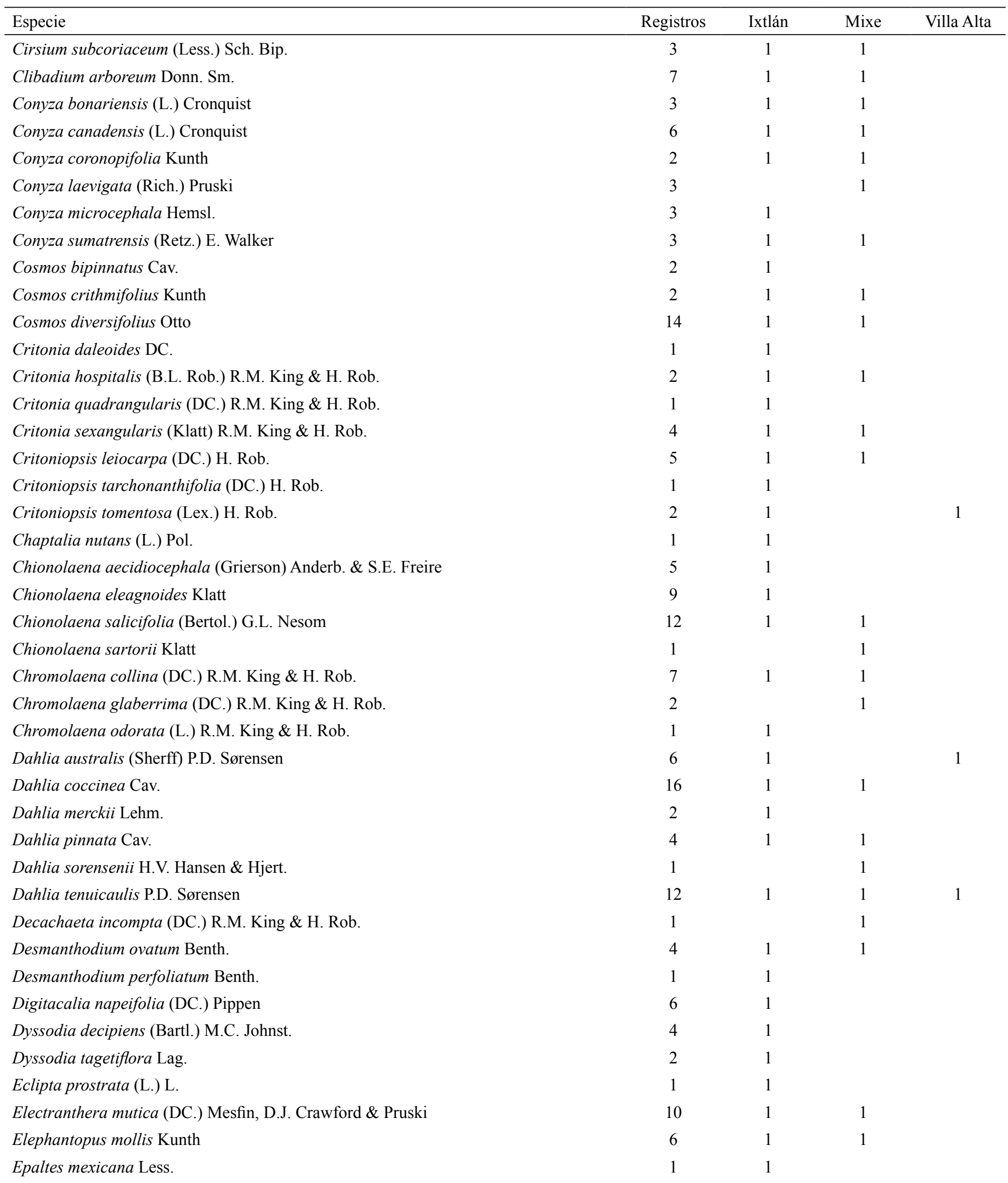


Apéndice. Continuación.

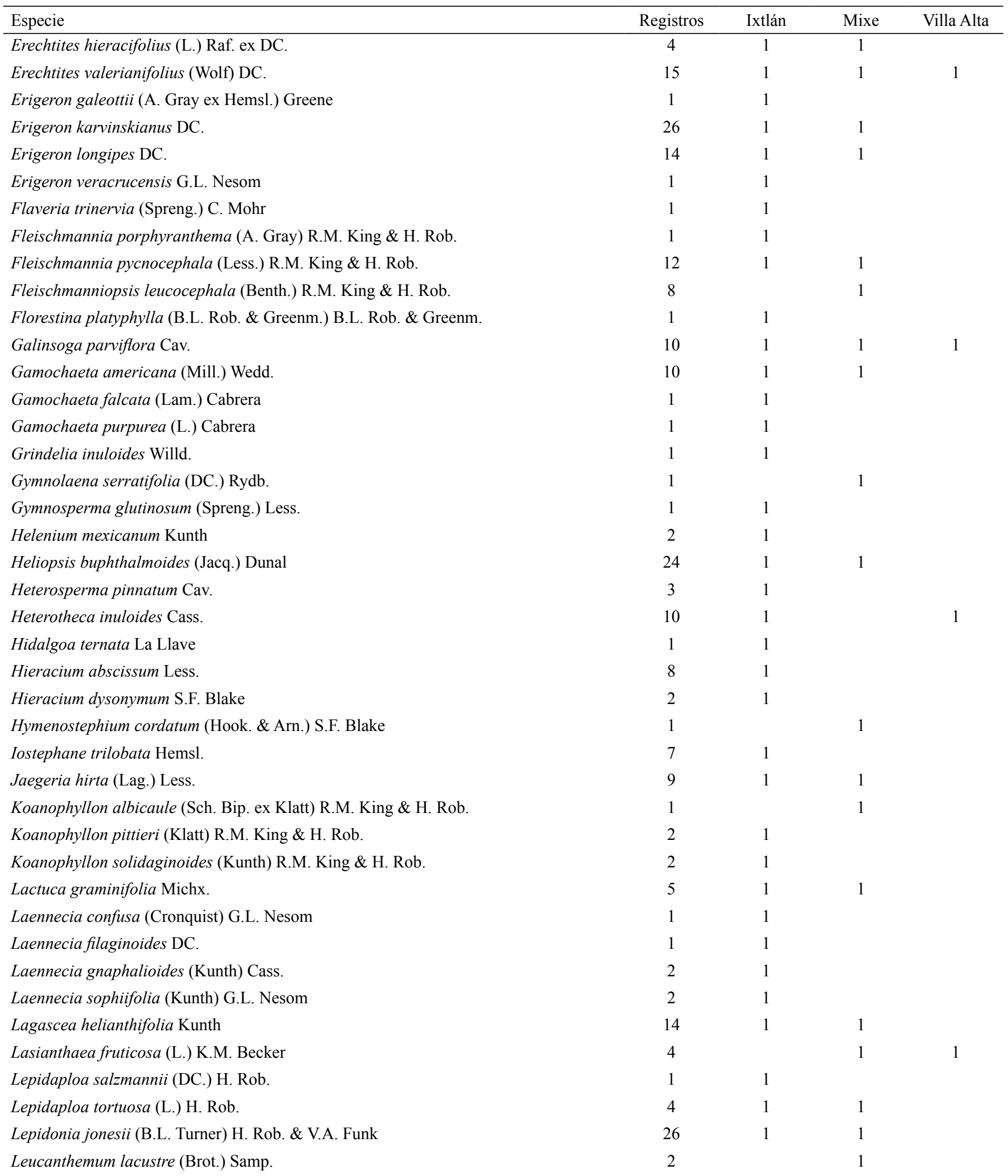


Apéndice. Continuación.

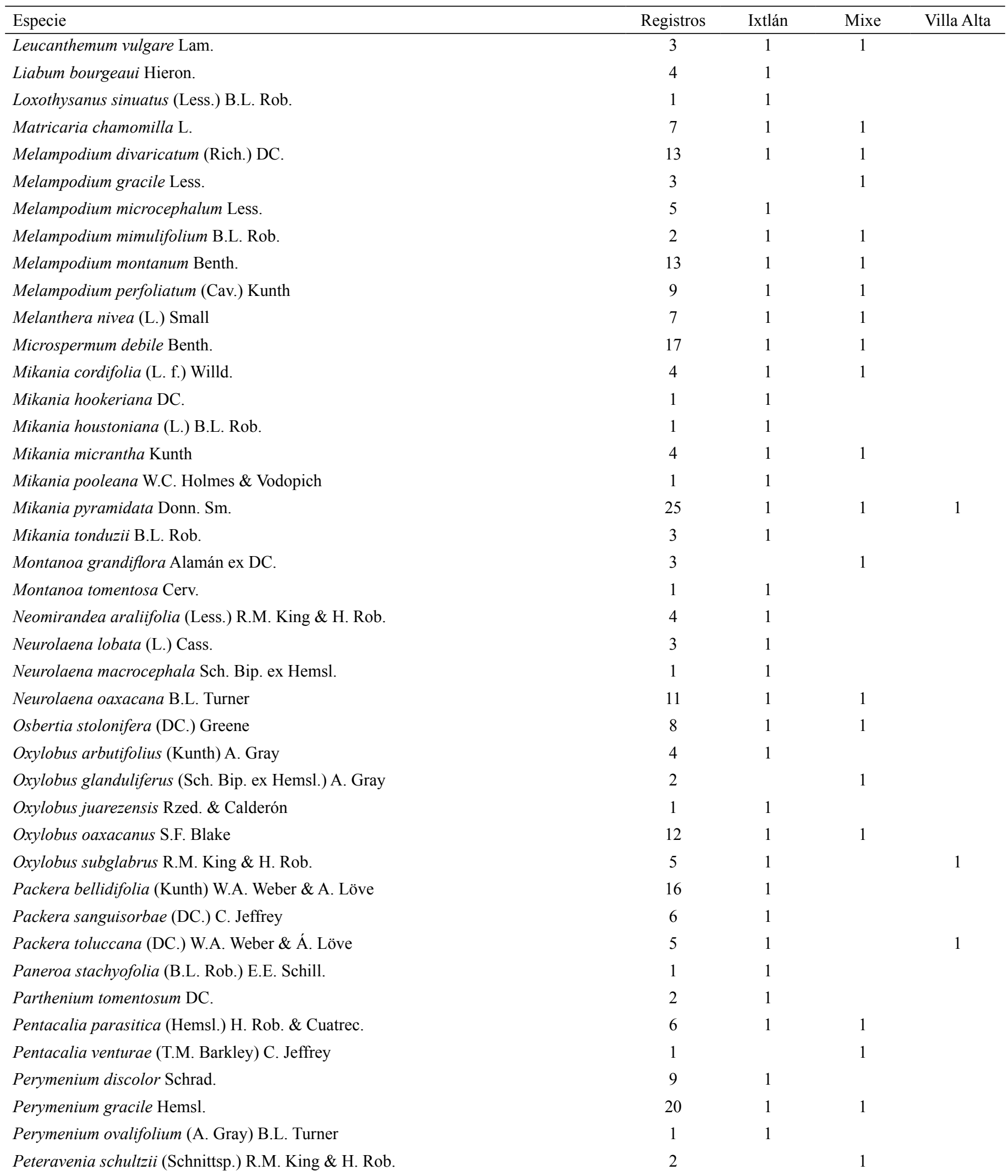


Apéndice. Continuación.

\begin{tabular}{|c|c|c|c|c|}
\hline Especie & Registros & Ixtlán & Mixe & Villa Alta \\
\hline Philactis zinnioides Schrad. & 7 & 1 & & 1 \\
\hline Pinaropappus roseus (Less.) Less. & 6 & 1 & & \\
\hline Piqueria trinervia Cav. & 5 & 1 & 1 & \\
\hline Pityopsis graminifolia (Michx.) Nutt. & 1 & 1 & & \\
\hline Pluchea salicifolia (Mill.) S.F. Blake & 2 & 1 & & \\
\hline Podachaenium eminens (Lag.) Sch. Bip. & 11 & 1 & 1 & 1 \\
\hline $\begin{array}{l}\text { Podachaenium pachyphyllum (Sch. Bip. ex Klatt) R.K. Jansen, N.A. Harriman \& } \\
\text { Urbatsch }\end{array}$ & 14 & & 1 & 1 \\
\hline Porophyllum linaria (Cav.) DC. & 7 & 1 & & \\
\hline Psacalium amplifolium (DC.) H. Rob. \& Brettell & 6 & 1 & 1 & \\
\hline Psacalium beamanii H. Rob. & 3 & 1 & & \\
\hline Psacalium cirsiifolium (Zucc.) H. Rob. \& Brettell & 1 & & 1 & \\
\hline Psacalium megaphyllum (B.L. Rob. \& Greenm.) Rydb. & 1 & & 1 & \\
\hline Psacalium peltatum (Kunth) Cass. & 4 & 1 & 1 & \\
\hline Pseudelephantopus spicatus (Juss. ex Aubl.) Rohr & 2 & 1 & 1 & \\
\hline Pseudognaphalium attenuatum (DC.) Anderb. & 1 & & 1 & \\
\hline Pseudognaphalium chartaceum (Greenm.) Anderb. & 3 & 1 & 1 & \\
\hline Pseudognaphalium elegans (Kunth) Kartesz & 1 & & & 1 \\
\hline Pseudognaphalium roseum (Kunth) Anderb. & 8 & 1 & 1 & \\
\hline Pseudognaphalium semilanatum (DC.) Anderb. & 2 & & 1 & \\
\hline Pseudognaphalium viscosum (Kunth) Anderb. & 3 & 1 & & \\
\hline Roldana angulifolia (DC.) H. Rob. \& Brettell & 3 & 1 & 1 & \\
\hline Roldana anisophylla (Klatt) Funston & 12 & 1 & 1 & \\
\hline Roldana aschenborniana (S. Schauer) H. Rob. \& Brettell & 7 & 1 & & \\
\hline Roldana barba-johannis (DC.) H. Rob. \& Brettell & 10 & 1 & & \\
\hline Roldana cordovensis (Hemsl.) H. Rob. \& Brettell & 1 & & 1 & \\
\hline Roldana jurgensenii (Hemsl.) H. Rob. \& Brettell & 1 & 1 & & \\
\hline Roldana lanicaulis (Greenm.) H. Rob. \& Brettell & 14 & 1 & 1 & \\
\hline Roldana lineolata (DC.) H. Rob. \& Brettell & 5 & 1 & 1 & \\
\hline Roldana oaxacana (Hemsl.) H. Rob. \& Brettell & 31 & 1 & 1 & \\
\hline Roldana sartorii (Sch. Bip. ex Hemsl.) H. Rob. \& Brettell & 4 & 1 & 1 & \\
\hline Roldana schaffneri (Sch. Bip. ex Klatt) H. Rob. \& Brettell & 3 & 1 & 1 & \\
\hline
\end{tabular}


Apéndice. Continuación.

\begin{tabular}{|c|c|c|c|c|}
\hline Especie & Registros & Ixtlán & Mixe & Villa Alta \\
\hline Rumfordia floribunda DC. & 9 & 1 & 1 & \\
\hline Sabazia multiradiata (Seaton) Longpre & 2 & 1 & 1 & \\
\hline Salmea scandens (L.) DC. & 4 & & 1 & \\
\hline Sanvitalia angustifolia Engelm. ex A. Gray & 1 & 1 & & \\
\hline Schistocarpha bicolor Less. & 10 & 1 & 1 & \\
\hline Schistocarpha pedicellata Klatt & 4 & 1 & 1 & \\
\hline Schistocarpha platyphylla Greenm. & 18 & 1 & 1 & 1 \\
\hline Schkuhria pinnata (Lam.) Kuntze & 4 & 1 & & \\
\hline Senecio bracteatus Klatt & 21 & 1 & & \\
\hline Senecio picridis S. Schauer & 10 & 1 & 1 & \\
\hline Senecio polypodioides (Greene) T. Durand \& B.D. Jacks. & 13 & 1 & & \\
\hline Sidneya pinnatilobata (Sch. Bip.) E.E. Schill. \& Panero & 1 & 1 & & \\
\hline Sigesbeckia jorullensis Kunth & 23 & 1 & 1 & \\
\hline Sigesbeckia repens B.L. Rob. \& Greenm. & 7 & 1 & & \\
\hline Simsia amplexicaulis (Cav.) Pers. & 1 & 1 & & \\
\hline Simsia sanguinea A. Gray & 1 & 1 & & \\
\hline Sinclairia deppeana (Less.) Rydb. & 15 & 1 & 1 & \\
\hline Stevia decumbens (B.L. Rob. \& Greenm.) Greene & 3 & 1 & & \\
\hline Stevia deltoidea Greene & 2 & 1 & & \\
\hline Stevia elatior Kunth & 6 & 1 & 1 & \\
\hline Stevia incognita Grashoff & 3 & 1 & & 1 \\
\hline Stevia jorullensis Kunth & 10 & 1 & 1 & \\
\hline Stevia latifolia Benth. & 1 & 1 & & \\
\hline Stevia lucida Lag. & 16 & 1 & & \\
\hline Stevia microchaeta Sch. Bip. & 57 & 1 & 1 & 1 \\
\hline Stevia monardifolia Kunth & 2 & 1 & & \\
\hline Stevia oligophylla Soejima \& Yahara & 1 & 1 & & \\
\hline Stevia origanoides Kunth & 2 & 1 & & \\
\hline Stevia ovata Willd. & 7 & 1 & 1 & 1 \\
\hline Stevia polycephala Bertol. & 8 & 1 & 1 & \\
\hline Stevia purpusii B.L. Rob. & 1 & 1 & & \\
\hline
\end{tabular}


Apéndice. Continuación.

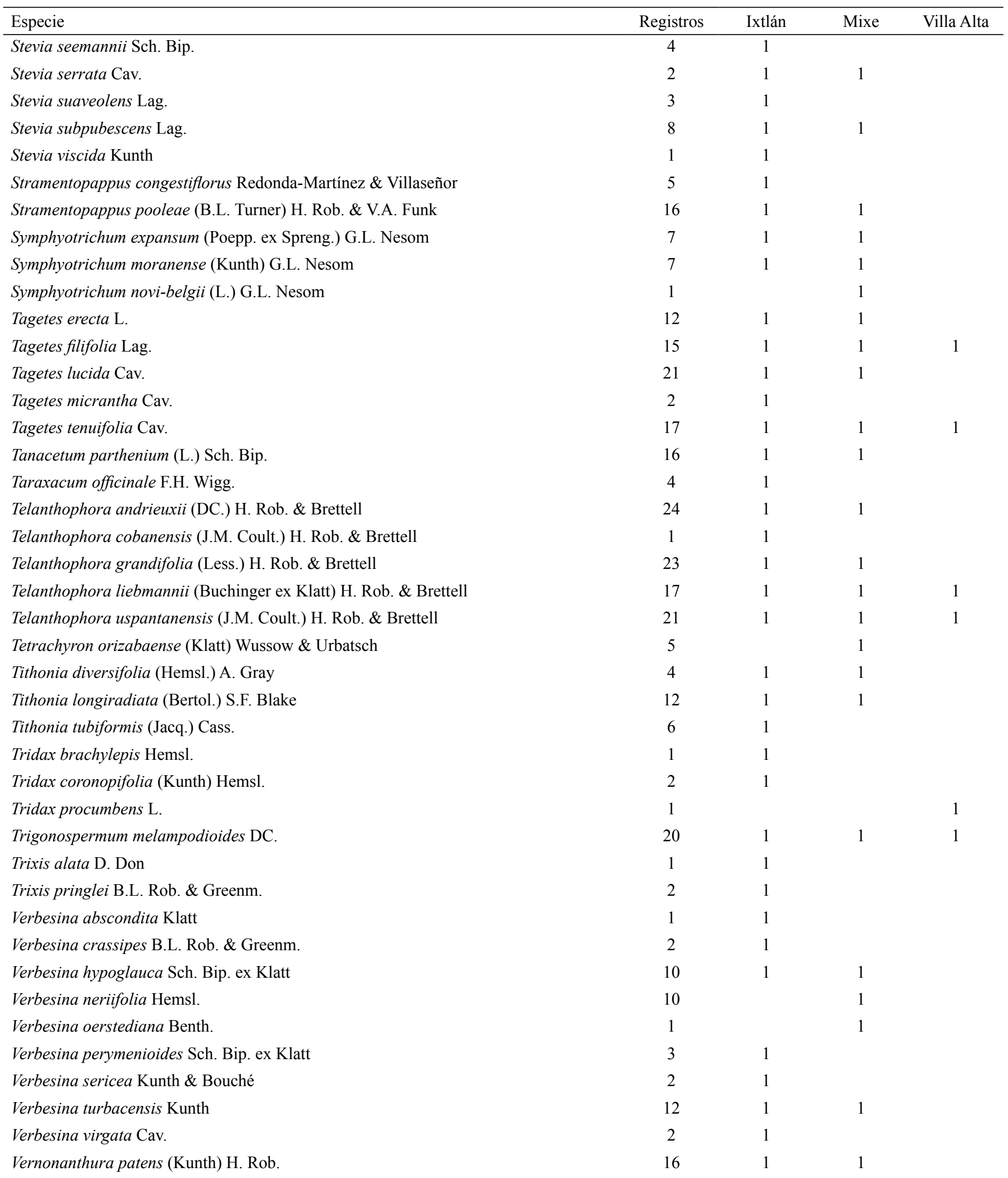


Apéndice. Continuación.

\begin{tabular}{|c|c|c|c|c|}
\hline Especie & Registros & Ixtlán & Mixe & Villa Alta \\
\hline Vernonia karvinskiana DC. & 24 & 1 & 1 & \\
\hline Xanthium strumarium L. & 1 & 1 & & \\
\hline Zexmenia serrata La Llave & 2 & 1 & & \\
\hline Zinnia elegans Jacq. & 3 & 1 & & \\
\hline
\end{tabular}

